\title{
Understanding Sensory Induced Hallucinations: From Neural Fields to Amplitude Equations
}

\author{
Rachel Nicks*, Abigail Cocks*, Daniele Avitabile ${ }^{\dagger}$, Alan Johnston ${ }^{\ddagger}$, and Stephen Coombes*
}

\begin{abstract}
Explorations of visual hallucinations, and in particular those of Billock and Tsou [Neural interactions between flicker-induced self-organized visual hallucinations and physical stimuli. Proceedings of the National Academy of Sciences, 104(20):8490-8495, 2007], show that annular rings with a background flicker can induce visual hallucinations in humans that take the form of radial fan shapes. The wellknown retino-cortical map tells us that the corresponding patterns of neural activity in the primary visual cortex for rings and arms in the retina are orthogonal stripe patterns. The implication is that cortical forcing by spatially periodic input can excite orthogonal modes of neural activity. Here we show that a simple scalar neural field model of primary visual cortex with state-dependent spatial forcing is capable of modelling this phenomenon. Moreover, we show that this occurs most robustly when the spatial forcing has a 2:1 resonance with modes that would otherwise be excited by a Turing instability. By utilising a weakly nonlinear multiple-scales analysis we determine the relevant amplitude equations for uncovering the parameter regimes which favour the excitation of patterns orthogonal to sensory drive. In combination with direct numerical simulations we use this approach to shed further light on the original psychophysical observations of Billock and Tsou.
\end{abstract}

Key words. Visual hallucinations, Neural field model, Spatially forced pattern forming system, Amplitude equations.

AMS subject classifications. 92C20, 35B36, 37L10.

1. Introduction. The story of spontaneous pattern formation in models of visual cortex is one that has attracted much attention since it was developed in the 1970s by Ermentrout and Cowan to explain drug induced geometric visual hallucinations [10]. These often take the form of lattice (a.k.a. honeycomb, grating, or chessboard), cobweb-like, tunnel (a.k.a. funnel, cone or vessel), and spiral patterns, as described in the experiments of Klüver [15] in which participants were given mescaline. When transformed from the retinocentric coordinates of the eye to the coordinates of the primary visual cortex (V1), these so-called Klüver form constants manifest as simple geometric planforms such as rolls, hexagons, squares, etc. [27]. It was the great insight of Ermentrout and Cowan that some of these could be generated via a Turing instability in a simple neural field model of V1. Neural fields are essentially continuum descriptions of cortical neural activity described by integro-differential equations. They are specified by a set of non-local spatial interaction kernels and nonlinear firing rate functions to describe the coarse grained activity of interacting excitatory and inhibitory neuronal populations, and for a recent review see [6]. Despite the difference in their mathematical form to many other pattern forming systems that arise in the modelling of physical systems, and in particular partial differential equations of reaction-diffusion type, they can be analysed using many of the same techniques. For example, a weakly nonlinear analysis can be used to derive

\footnotetext{
*School of Mathematical Sciences, University of Nottingham, UK. (rachel.nicks@nottingham.ac.uk, abigail.cocks@nottingham.ac.uk, stephen.coombes@nottingham.ac.uk).

${ }^{\dagger}$ Department of Mathematics, Vrije Universiteit Amsterdam, Netherlands. (d.avitabile@vu.nl).

${ }^{\ddagger}$ School of Psychology, University of Nottingham, UK. (alan.johnston@nottingham.ac.uk).
} 
the amplitude equations for patterns emerging beyond the point of a Turing instability [30, 8]. More recently an extension of the original work by Ermentrout and Cowan has been developed by Bressloff et al. [4] to describe the dynamics of orientation selective cells. This more biologically realistic neural field model includes anisotropic lateral connections that only connect distal elements with the same orientation preference along the direction of their (common) orientation preference. Interestingly this model can generate representatives of all the Klüver form constants. Nonetheless both this and the original model of Ermentrout and Cowan have a focus on spontaneous pattern formation that is induced by changes of parameters intrinsic to the models, rather than by external drive. However, it is particularly important to address this when trying to understand the mechanisms of sensory induced illusions and hallucinations in response to the presentation of either static or dynamic visual input. An example of the former is the flickering wheel illusion whereby a static wheel stimulus, with $30-40$ spokes, is experienced as flickering when viewed in the visual periphery [29]. A perhaps more well known sensory induced percept is that of illusory rotational motion experienced when looking at the rotating snakes image [5] (and for an example visit [14]). Interestingly, since the work of MacKay in the 1950s it is well known that relatively simple patterns of regular stimuli, such as radial lines or concentric rings, are enough to induce illusory motion at right angles to those of the stimulus pattern [18]. Many of these phenomenon are amenable to further study using the tools of psychophysics. A case in point, and the focus of the theoretical study presented here, are the visual hallucinations reported in the work of Billock and Tsou [3]. These authors tried to induce certain geometric hallucinations by biasing them with an appropriate visual stimuli from a flickering monitor. For example, a set of centrally presented concentric rings was expected to induce a hallucination of circle in the surround. Instead, and to their surprise, they found that fan-shaped patterns were perceived in the surround (and a complementary pattern of concentric ring circles in the surround for radial patterns in the centre). The retinocortical map, mentioned above, tells us that the corresponding patterns of neural activity in the primary visual cortex for rings and arms in the retina are orthogonal stripe patterns. The implication of the psychophysical experiments of Billock and Tsou is that cortical forcing by spatially periodic input can excite orthogonal modes of neural activity. Thus, a natural question arises as to whether there is a minimal model of visual cortex with external drive capable of supporting this observed orthogonal response and does it require a departure from existing neural field models. In short the answer is that standard neural field models with a state-dependent drive are sufficient. Although the orthogonal response property may seem somewhat surprising from an experimental perspective, relatively recent theoretical studies of the spatially forced Swift-Hohenberg equation have shown that under certain mild conditions orthogonal responses are robust [20]. Here we adapt and develop the techniques originally developed for analysing spatially forced partial differential equation models to nonlocal neural fields, and use these to uncover the parameter windows that robustly reproduce orthogonal responses to spatially periodic forcing. In doing so we highlight the potential mechanisms that can underpin the original psychophysical observations of Billock and Tsou.

In $\S 2$ we describe in more detail the psychophysical experiments of Billock and Tsou as well as introduce a simple neural field model with state-dependent drive that will subsequently be shown to be a minimal model for their observations. The key mechanism for the success of the model is the combination of a Turing instability and a 2:1 resonance arising between the 
spatial scale of the periodic forcing and that of the emergent Turing pattern. An important parameter of the model is then the spatial frequency mismatch between these two scales. The Turing and resonance effects are analysed in $\S 3$. In $\S 4$ we develop a weakly nonlinear analysis, valid for weak forcing in the neighbourhood of a Turing instability, and derive equations governing the amplitude of emergent planforms. These in turn are analysed using bifurcation theory to uncover appropriate parameter choices (in the strength of forcing, the frequency mismatch, and shape of the nonlinear firing rate) to generate an orthogonal response. This theoretical work is complemented in $\S 5$ with direct numerical simulations, for both globally periodic and spatially structured patterns of drive, to both confirm our analysis and make a more concrete connection with psychophysical observations. Finally in $\S 6$ we discuss the main results of our paper and highlight areas for future work.

2. Psychophysics and a model. Surprisingly little is known about the interactions between sensory driven and self organised cortical activity. Billock and Tsou have worked to address this deficit by probing the link between natural visual perception and the geometric hallucinations that can be induced by the presentation of certain regular spatio-temporal patterns. In a set of human psychophysical experiments using a flickering monitor (at $10-15 \mathrm{~Hz}$ in a dark room where the stimuli was $1 / 10$ th to $1 / 3$ rd of the flickered area) they found the surprising result that biasing stimuli could provoke an orthogonal response. For example, if the area around a small fan shape is flickered, subjects report seeing illusory circular patterns. This is considered an orthogonal response since the corresponding patterns of activity in V1 are stripes of activity oriented at right angles to each other. This latter result stems from the well known retino-cortical map that maps radial arms in the visual field to horizontal stripes of activity in V1, and concentric rings to vertical stripes (with respect to a ventral-dorsal axis). To a first approximation this map (away from the fovea) is often approximated by a quasi-conformal dipole map [2] that would map spiral arms in retinal coordinates to oblique stripes in cortical coordinates, as illustrated in Fig. 1. The cortical map can also be thought of as a spherical map in the eye stretched along the optical axis and viewed from the side [13]. One might say that if the image of a circle opposed by a radial arm is considered on the retina then it is locally orthogonal, whereas if the corresponding cortical activity is considered then it is globally orthogonal. Billock and Tsou also reported similar orthogonal responses in three other scenarios: i) if the area around a circular pattern is flickered, an illusory rotating fan shape is perceived (and if the circles are flickering too, the rotating fan shape extends through the physical circles), ii) if a biasing pattern of peripheral radial arms is presented then central (tightly packed) rings are perceived, and iii) a rotating petal-like pattern often appears in the flickering central area in response to a peripheral set of biasing concentric rings. These types of hallucinatory percepts are all illustrated in Fig. 2. In all cases of perceived rotation (typically between 0.75 and 1.3 revolutions per second) the direction of rotation is arbitrary and subject to reversal.

A major conclusion of Billock and Tsou is that the pattern of sensory induced hallucinations in their psychophysical experiments reflects the same cortical properties, including local connectivity and lateral inhibition within a retinotopic map in V1, that shape routine visual processing. Given the success of neural field models in describing drug-induced (spontaneous) hallucinations in V1, it is thus natural to see if they are also capable of explaining the op- 

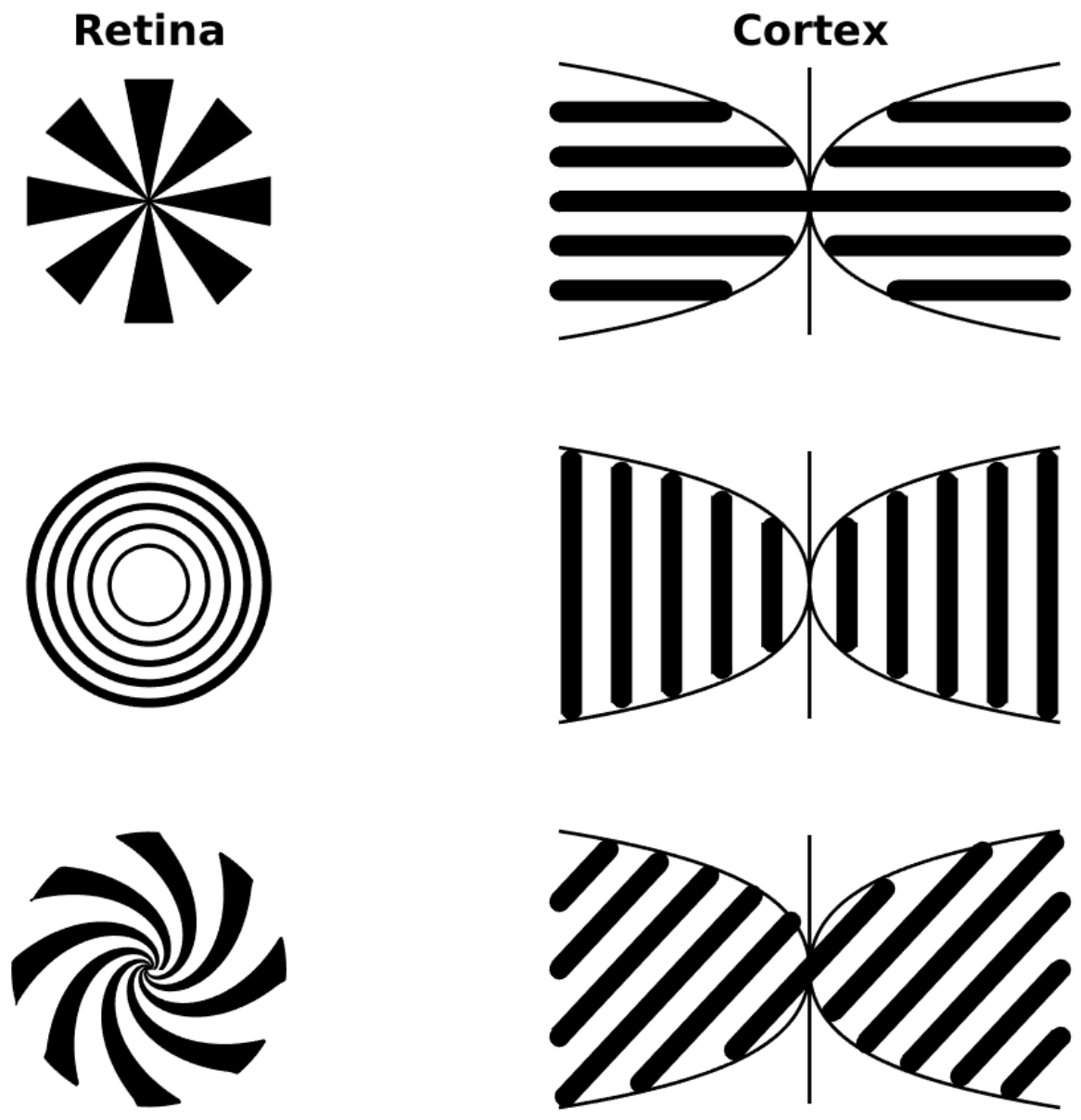

Figure 1: An illustration of the retino-cortical map that takes points of stimuli on the retina to points in V1 (left and right primary visual cortex), showing how radial arms, rings, and spirals on the retina transform to oriented stripes on V1.

ponency in these flicker-induced visual phenomena. To this end we now consider a minimal model of V1 with the inclusion of a forcing term to mimic sensory input to the system.

Here we consider a simple neural field model for the evolution of synaptic activity in an effective single population with adaptation. The different effects of excitatory and inhibitory interactions are encoded in a single kernel whose sign indicates whether an interaction is excitatory (positive) or inhibitory (negative). We do this for mathematical convenience though stress that the approach developed for model analysis is equally applicable to treating populations of interacting excitatory and inhibitory neuronal populations separately. The inclusion of adaptation means that the model is more realistic, in the sense that this gives a phenomenological description of metabolic processes that lead to fatigue. It also provides a well known route to dynamic instabilities leading to the formation of travelling waves. The latter are expected to be a key requirement for illusory motion. We shall also work with a kernel 

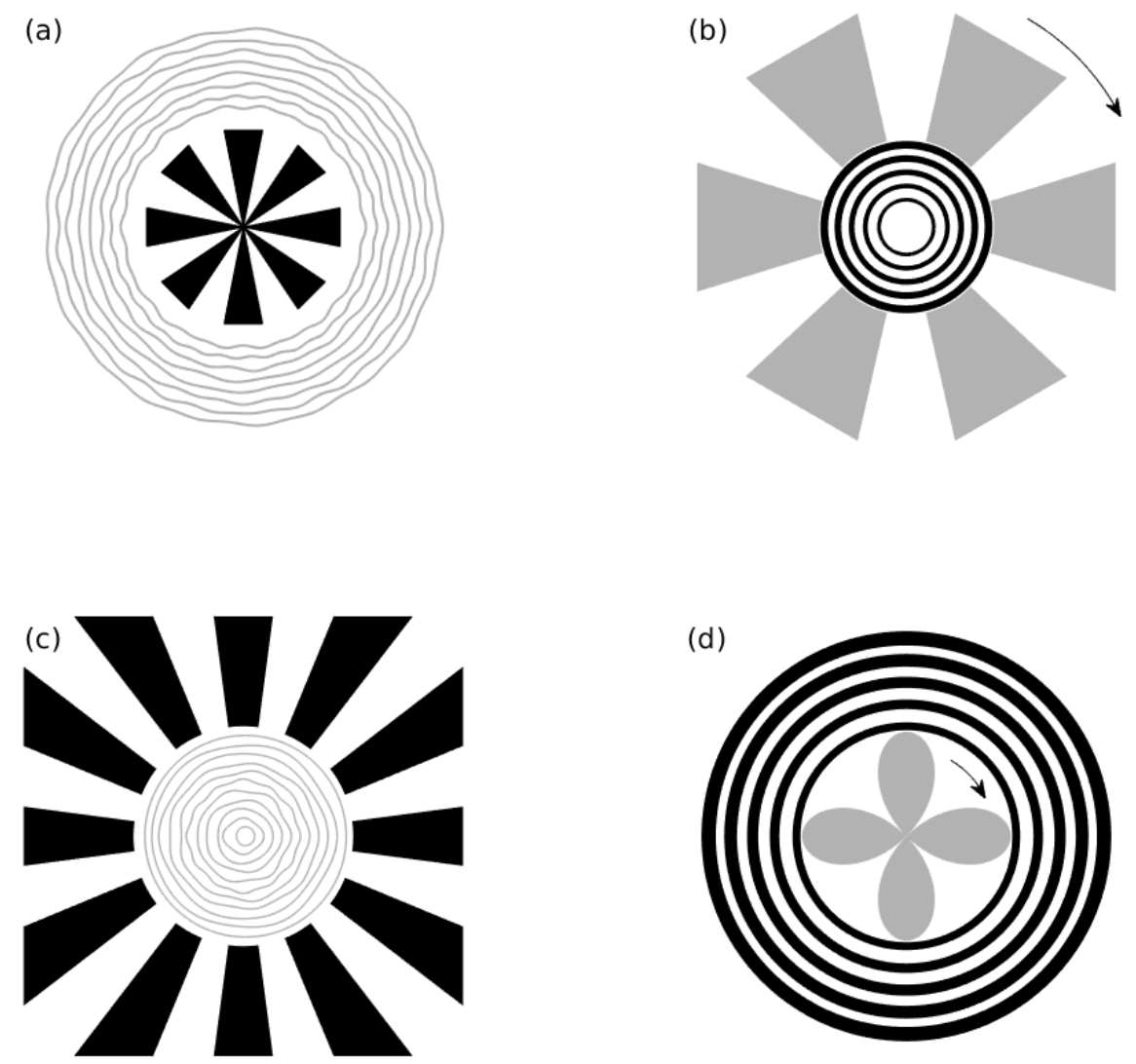

Figure 2: An illustration of the biasing stimuli (black) and hallucinatory percepts (grey) as reported by Billock and Tsou and redrawn from [3]. (a) If the area around a small fan shape is flickered, subjects report seeing illusory circular patterns, (b) if the area around a circular pattern is flickered, an illusory rotating fan shape is perceived, (c) if a biasing pattern of peripheral radial arms is presented then central rings are perceived, and (d) a rotating petal-like pattern often appears in the flickering central area in response to a peripheral set of biasing concentric rings. The arrows indicate perceived rotation.

that describes a tissue with short-range excitation and long-range inhibition, which is well known for its pattern forming properties [1,8]. Given the phenomenological nature of neural field models we adopt a similar approach for the modelling of visual input to V1. From a biological perspective cells in V1 would be driven by synaptic currents, and these in turn would be mediated by conductance changes arising from afferent inputs. These currents have a simple ohmic form that multiplies the voltage of the post-synaptic neuron with that of the conductance change. Thus the input signal is mixed with the state of the neuron. We shall be careful to carry over this important effect into our phenomenological model of drive.

Introducing the vector field $(u, a)$ we write our neural field model with drive in the succinct 
form

$$
\begin{aligned}
\frac{\partial u}{\partial t} & =-u+w \otimes f(u)-g a+\gamma u I, \\
\tau_{a} \frac{\partial a}{\partial t} & =u-a .
\end{aligned}
$$

Here $u$ is a scalar field representing neural activity and $a$ is a scalar field representing a negative feedback adaptation variable. The symbol $\otimes$ denotes a spatial convolution, and $f$ is a nonlinear firing rate (typically sigmoidal in shape). The kernel $w$ is chosen to encode the spatial interactions between points in the tissue (taken to be translationally and rotationally invariant). The parameter $g \geq 0$ represents the strength of the adaptive feedback and $\tau_{a}>0$ sets the relative time-scale. The external input is described by $I$ and we allow for a simple form of mixing by including a multiplication with the state $u$. The strength of forcing is described by $\gamma \in \mathbb{R}$. We could, of course, have placed the forcing $I$ inside the firing rate $f$. However, a nonlinear Taylor expansion would expose multiplicative terms, and to keep the analysis in this paper as uncomplicated as possible we prefer instead the choice made, though emphasise that the analysis to follow is easily adapted to this case (albeit at the expense of slightly more complicated calculations). The model described by (2.1) and (2.2) can be posed in a variety of spatial domains. In this paper we shall focus on a planar system so that $(u, a, I)=(u(\mathbf{r}, t), a(\mathbf{r}, t), I(\mathbf{r}, t))$ with $\mathbf{r}=(x, y) \in \mathbb{R}^{2}$ and $t>0$, so that

$$
[w \otimes f(u)](\mathbf{r}, t)=\int_{\mathbb{R}^{2}} \mathrm{~d} \mathbf{r}^{\prime} w\left(\mathbf{r}-\mathbf{r}^{\prime}\right) f\left(u\left(\mathbf{r}^{\prime}, t\right)\right) .
$$

Here the kernel function $w$ depends only upon distance so that $w(\mathbf{r})=w(r)$, where $r=|\mathbf{r}|$. For concreteness we will work with the rotationally symmetric Wizard hat function (although the theory we develop is ambivalent to the particular choice of Mexican-hat style function):

$$
w(r)=A \mathrm{e}^{-r / \sigma}-\mathrm{e}^{-r}, \quad A>1, \sigma<1 .
$$

Moreover, for later convenience and without undue restriction, we impose the balance condition $\int_{\mathbb{R}^{2}} \mathrm{~d} \mathbf{r} w(|\mathbf{r}|)=0$, which is achieved when $A=\sigma^{-2}$. The firing rate function is chosen as a sigmoid with a threshold $h$ and steepness parameter $\mu$ :

$$
f(u)=\frac{1}{1+\mathrm{e}^{-\mu(u-h)}} .
$$

Finally the model is completed with the choice of drive $I(\mathbf{r}, t)$. Since we are primarily interested in the mechanisms that underly an orthogonal response we shall develop theory for the case that this is a simple spatial pattern of stripes in the $x$-direction with a spatial forcing wavenumber $k_{f}$ and write $I(\mathbf{r}, t)=\cos \left(k_{f} x\right)$. Our interest is in the development of striped patterns in neural activity along the $y$-direction.

3. Turing instability and resonances. The use of a Turing instability analysis to understand pattern formation in neural fields is exemplified by the work of Ermentrout and Cowan [10]. In their original work the emphasis was on spontaneous pattern formation in the absence of external input, and they highlighted that a mixture of short-range excitation and 
long-range inhibition was key for the emergence of global patterning. Perhaps surprisingly, the study of forced neural fields has received relatively little attention in the mathematical literature, one exception being the work of Rule et al. [26] which considers spatially homogeneous, time periodic forcing and shows (using symmetric bifurcation theory) that stripes occur at high frequency forcing (2:1 resonance) and hexagons at low. In the current work we consider spatially inhomogeneous forcing, namely forcing with stripes. To gain insight into the effects of spatial forcing it is timely to adapt recent results developed for the Swift-Hohenberg equation [20]. Here we first review the spontaneous patterning behaviour of the neural field model without drive and then show how resonant patterns can emerge when spatially periodic drive is introduced.

3.1. Patterning in the absence of drive. First consider the case with no drive, namely with $\gamma=0$ and write the model (2.1)-(2.2) in the integro-differential form

$$
\frac{\partial u}{\partial t}=-u+w \otimes f(u)-g \eta * u
$$

Here we have exploited the linearity of (2.2) to integrate the equations of motion for $a$ (assuming vanishing initial data) and introduced the temporal convolution

$$
[\eta * u](\mathbf{r}, t)=\int_{-\infty}^{t} \mathrm{~d} t^{\prime} \eta\left(t-t^{\prime}\right) u\left(\mathbf{r}, t^{\prime}\right), \quad \eta(t)=\frac{1}{\tau_{a}} \mathrm{e}^{-t / \tau_{a}} H(t),
$$

where $H$ is a Heaviside step function.

It is convenient to introduce the Fourier transform of $w$ as $\widehat{w}$ in the form

$$
\widehat{w}(\mathbf{k})=\int_{\mathbb{R}^{2}} \mathrm{~d} \mathbf{r} w(\mathbf{r}) \mathrm{e}^{-i \mathbf{k} \cdot \mathbf{r}}, \quad \mathbf{k} \in \mathbb{R}^{2},
$$

and the Laplace transform of $\eta$ as $\widetilde{\eta}$ in the form

$$
\widetilde{\eta}(\lambda)=\int_{0}^{\infty} \mathrm{d} t \eta(t) \mathrm{e}^{-\lambda t}, \quad \lambda \in \mathbb{C} .
$$

For a rotationally symmetric kernel we also have that $\widehat{w}(\mathbf{k})=\widehat{w}(k)$, where $k=|\mathbf{k}|$. For the choice (2.4) we have the explicit result that

$$
\widehat{w}(k)=2 \pi\left[\frac{A}{\sigma\left(\sigma^{-2}+k^{2}\right)^{3 / 2}}-\frac{1}{\left(1+k^{2}\right)^{3 / 2}}\right],
$$

and for $\widetilde{\eta}$ we have that

$$
\widetilde{\eta}(\lambda)=\frac{1}{1+\lambda \tau_{a}} .
$$

The homogeneous steady state $(u(\mathbf{r}, t), a(\mathbf{r}, t))=\left(u_{0}, a_{0}\right)$ of the neural field model is then given by $a_{0}=u_{0}$ with $u_{0}=\widehat{w}(0) f\left(u_{0}\right) /(1+g \widetilde{\eta}(0))$. For a balanced kernel $\widehat{w}(0)=0$ and we have that $\left(u_{0}, a_{0}\right)=(0,0)$ for all model parameter choices (when $\left.\gamma=0\right)$. Linearising around 
the homogeneous steady state by writing $u(\mathbf{r}, t)=u_{0}+\epsilon \delta u(\mathbf{r}, t)$, for some small amplitude $|\epsilon| \ll 1$, and expanding to first order gives the evolution for the perturbations as

$$
\frac{\partial}{\partial t} \delta u=-\delta u+f^{\prime}\left(u_{0}\right) w \otimes \delta u-g \eta * \delta u .
$$

We note that for the choice $(2.5)$ we have $f^{\prime}(u)=\mu f(u)(1-f(u))$. Equation (3.7) has separable solutions of the form $\delta u(\mathbf{r}, t)=\mathrm{e}^{\lambda t} \mathrm{e}^{i \mathbf{k} \cdot \mathbf{r}}$ where the dispersion relation between $\lambda$ and $|\mathbf{k}|$ can be written implicitly in the form $\mathcal{E}(\lambda, k)=0$ with

$$
\mathcal{E}(\lambda, k)=1+\lambda+g \widetilde{\eta}(\lambda)-f^{\prime}\left(u_{0}\right) \widehat{w}(k) .
$$

To obtain the above we have used the result that $w \otimes \mathrm{e}^{i \mathbf{k} \cdot \mathbf{r}}=\widehat{w}(\mathbf{k}) \mathrm{e}^{i \mathbf{k} \cdot \mathbf{r}}$ and $\eta * \mathrm{e}^{\lambda t}=\widetilde{\eta}(\lambda) \mathrm{e}^{\lambda t}$. For $g=0$ (no adaptation) then the spectrum is given explicitly by

$$
\lambda=-1+f^{\prime}\left(u_{0}\right) \widehat{w}(k) .
$$

Since $w$ is translationally invariant then $\widehat{w}$ is real and we see that in this case $\lambda \in \mathbb{R}$. A static Turing instability (to a purely spatially periodic time-independent pattern) is then possible, with the bifurcation condition being $\widehat{w}\left(k_{0}\right)=1 / f^{\prime}\left(u_{0}\right)$. Here $k_{0}>0$ is the point at which $\widehat{w}(k)$ has a local maxima (namely $\widehat{w}\left(k_{0}\right)=\max \widehat{w}(k)$ ). Note that any direction on a circle of wavevectors of magnitude $|\mathbf{k}|=k_{0}$ can be excited. When $g>0$ it is possible that $\lambda$ can become complex. After decomposing $\lambda=\nu+i \omega$, and then equating real and imaginary parts of (3.8) it can be shown that the spectrum lies on the curve given by

$$
\tau_{a}^{2}\left(\nu^{2}+\omega^{2}\right)+2 \tau_{a} \nu=\tau_{a} g-1,
$$

and to the left of the line,

$$
\nu=\frac{-\left(1+\tau_{a}-\tau_{a} f^{\prime}\left(u_{0}\right) \widehat{w}\left(k_{0}\right)\right)}{2 \tau_{a}} .
$$

Thus for $g>0$ a Turing instability to a dynamic (time-dependent) pattern $(\omega \neq 0)$ will occur when $\widehat{w}\left(k_{0}\right)=\left(1+\tau_{a}\right) /\left(\tau_{a} f^{\prime}\left(u_{0}\right)\right)$ for $\tau_{a} g>1$ and $g>f^{\prime}\left(u_{0}\right) \widehat{w}\left(k_{0}\right)-1$ (and the latter condition excludes the possibility of a static bifurcation). The emergent frequency of oscillation is $\omega_{c}=\sqrt{\tau_{a} g-1} / \tau_{a}$. We note that the conditions for static and dynamic Turing instabilities given here agree those in [7] since the model equations only differ in the placement of the nonlinear firing rate.

3.2. Resonant patterns in the presence of drive. The periodic forcing of pattern forming system can lead to novel behaviours as well as frequency or wavenumber locking. The mathematical study of periodic temporal forcing is more well developed than its spatial structured counterpart, and it is well known that this can lead to $n: 1$ resonances in both ordinary differential systems with a Hopf bifurcation [11] and partial differential equations [17]. The mathematical study of spatially forced pattern forming systems is relatively underdeveloped compared to that of temporal forcing, with an exception being the work of Manor et al. [19]. In this and follow up work [20,21, 22], these authors consider idealised pattern forming systems of Swift-Hohenberg type poised near Turing instability to a pattern with a wavenumber 
$k_{0}$ with weak spatial periodic spatial forcing at wavenumber $k_{f}$. They show that if $k_{f}$ is close to $2 k_{0}$ then stable resonant stripes can be formed. Importantly, they also establish that if the mismatch between $k_{f}$ and $k_{0}$ is high, then a locked pattern can still develop albeit with a wavevector component perpendicular to the forcing direction. Given that this is one of the major properties of the psychophysical experiments of Billock and Tsou that we are seeking to understand, it is natural to see if the corresponding phenomenon can arise in a neural field model. To first probe whether resonances arise naturally in a neural field model with forcing we note that this question does not require a treatment in two spatial dimensions. Given that resonances can be explored in a one dimensional setting we consider here the neural field model (3.1) posed on the real line (rather than the plane). This is useful not only for simplifying calculations, but also for setting the scene for the analysis of the fully two-dimensional model that we shall present next in $\S 4$. Although the psychophysical experiments of Billock and Tsou involve temporal flicker we will show below that it is not strictly necessary to include this to generate opponent patterns.

In the presence of spatially periodic drive the model equation is

$$
\frac{\partial u}{\partial t}=-u+w \otimes f(u)-g \eta * u+\gamma u \cos \left(k_{f} x\right) .
$$

We consider a scalar field $u=u(x, t)$, with $x \in \mathbb{R}$ and $t>0$, governed by (3.12) with $\gamma \neq 0$. For simplicity we drop the treatment of adaptation for now and set $g=0$. From now on we will assume that the forcing wavenumber $k_{f}$ is approximately a multiple of $k_{0}$, so that $k_{f} \approx n k_{0}, n \in \mathbb{Z}$ and introduce a mismatch parameter $v$

$$
v=k_{0}-k_{f} / n \text {. }
$$

The value of $n$ can be used to describe an $n: 1$ resonance. If the system is poised at a static Turing instability to a pattern with wavenumber $k_{0}$ and the forcing is weak $(|\gamma| \ll 1)$ then it is natural to consider a multiple-scales analysis to understand the response properties of the driven system. We assume that the small detuning can be scaled as $v=\epsilon c$ for a small parameter $\epsilon$. We then define new scaled variables $\chi=\epsilon x$ and $\tau=\epsilon^{2} t$ and consider power series expansions for $u$ and $\gamma$ as

$$
\begin{aligned}
& u=u_{0}+\epsilon u_{1}+\epsilon^{2} u_{2}+\epsilon^{3} u_{3}+\ldots, \\
& \gamma=\epsilon \gamma_{1}+\epsilon^{2} \gamma_{2}+\epsilon^{3} \gamma_{3}+\ldots
\end{aligned}
$$

with, as yet, unknown functions $u_{\alpha}=u_{\alpha}(x, t, \chi, \tau), \alpha=1,2,3, \ldots$ Further, we substitute the firing rate function $f$ by its Taylor series expansion $f(u)=f\left(u_{0}\right)+\beta_{1}\left(u-u_{0}\right)+\beta_{2}\left(u-u_{0}\right)^{2}+$ $\beta_{3}\left(u-u_{0}\right)^{3}+\ldots$, where $\beta_{2}=f^{\prime \prime}\left(u_{0}\right) / 2, \beta_{3}=f^{\prime \prime \prime}\left(u_{0}\right) / 6$, and we treat $\beta_{1}$ as a bifurcation parameter and write $\beta_{1}=\beta_{c}+\epsilon^{2} \delta$ where $\beta_{c}=f^{\prime}\left(u_{0}\right)$ subject to $\beta_{c}=1 / \widehat{w}\left(k_{0}\right)$ (the static Turing bifurcation condition). A further Taylor series expansion of the functions $u_{\alpha}$ as

$$
\begin{aligned}
& u_{\alpha}\left(y, s, \epsilon y, \epsilon^{2} s\right)=u_{\alpha}\left(y, s, \chi+\epsilon(y-x), \epsilon^{2} s\right) \\
& \simeq u_{\alpha}(y, s, \chi, \tau)+\epsilon(y-x) \frac{\partial}{\partial \chi} u_{\alpha}(y, s, \chi, \tau)+\epsilon^{2} \frac{1}{2}(y-x)^{2} \frac{\partial^{2}}{\partial \chi^{2}} u_{\alpha}(y, s, \chi, \tau)+O\left(\epsilon^{3}\right)
\end{aligned}
$$


facilitates an evaluation of the spatial convolution in (3.12). Balancing terms at powers of $\epsilon$ in (3.12) yields a hierarchy of equations as

$$
\begin{aligned}
u_{0}= & M_{0}\left(f\left(u_{0}\right)\right), \\
u_{1}= & M_{0}\left(\beta_{c} u_{1}\right)+\gamma_{1} u_{0} \cos \left(k_{f} x\right), \\
u_{2}= & M_{0}\left(\beta_{c} u_{2}+\beta_{2} u_{1}^{2}\right)+M_{1}\left(\beta_{c} u_{1}\right)+\left(\gamma_{1} u_{1}+\gamma_{2} u_{0}\right) \cos \left(k_{f} x\right), \\
\frac{\partial u_{1}}{\partial \tau}+u_{3}= & M_{0}\left(\beta_{c} u_{3}+\delta u_{1}+2 \beta_{2} u_{1} u_{2}+\beta_{3} u_{1}^{3}\right)+M_{1}\left(\beta_{c} u_{2}+\beta_{2} u_{1}^{2}\right) \\
& +M_{2}\left(\beta_{c} u_{1}\right)+\left(\gamma_{1} u_{2}+\gamma_{2} u_{1}+\gamma_{3} u_{0}\right) \cos \left(k_{f} x\right),
\end{aligned}
$$

where the linear operators $M_{\alpha}$ are given by $M_{0}=w \otimes, M_{1}=W^{x} \otimes \partial_{\chi}$, and $M_{2}=\frac{1}{2} W^{x x} \otimes \partial_{\chi \chi}$. Here we have introduced the new kernels $W^{x}(x)=-w(|x|) \cdot x$ and $W^{x x}(x)=w(|x|) \cdot x^{2}$. One can see that each equation in the hierarchy above contains terms of the asymptotic expansion of $u$ only of the same order or lower. This means that we can start from the first equation and systematically solve for $u_{\alpha}$. In fact, if we set $\mathcal{L}=-1+\beta_{c} w \otimes$ the system (3.18)-(3.20) has the general form $\mathcal{L} u_{\alpha}=g_{\alpha}\left(u_{1}, u_{2}, \ldots, u_{\alpha-1}\right)$ and the right-hand side $g_{\alpha}$ will always contain known quantities. The first equation (3.17) in the hierarchy fixes the steady state $u_{0}$. By choosing a balanced kernel we have $u_{0}=0$. Note that in one dimension the balance condition $\int_{-\infty}^{\infty} w(|x|) \mathrm{d} x=0$ for the kernel (2.4) is achieved when $A=\sigma^{-1}$. In this case we also have

$$
\widehat{w}(k)=2 \int_{0}^{\infty} w(x) \mathrm{e}^{-i k x} \mathrm{~d} x=2\left[\frac{1}{1+\sigma^{2} k^{2}}-\frac{1}{1+k^{2}}\right] .
$$

The second equation (3.18) is linear with solutions $u_{1}=A(\chi, \tau) \mathrm{e}^{i k_{0} x}+$ c.c. (where $k_{0}$ is the critical wavenumber at the static bifurcation). Hence the null space of $\mathcal{L}$ is spanned by $\mathrm{e}^{ \pm i k_{0} x}$. A dynamical equation for the complex amplitude $A(\chi, \tau)$ can be obtained by deriving solvability conditions for the higher-order equations, a method known as the Fredholm alternative.

We define the inner product of two periodic functions (with periodicity $2 \pi / k_{0}$ ) as

$$
\langle U, V\rangle=\frac{k_{0}}{2 \pi} \int_{0}^{\frac{2 \pi}{k_{0}}} U^{*}(x) V(x) \mathrm{d} x .
$$

For all $u \in \operatorname{ker} \mathcal{L}^{\dagger}$ then $\left\langle u, g_{\alpha}\right\rangle=\left\langle u, \mathcal{L} u_{\alpha}\right\rangle=\left\langle\mathcal{L}^{\dagger} u, u_{\alpha}\right\rangle=0$ where $\mathcal{L}^{\dagger}$ is the adjoint of $\mathcal{L}$. It is easy to establish that $\mathcal{L}$ is self-adjoint so that the set of solvability conditions are $\left\langle\mathrm{e}^{ \pm i k_{0} x}, g_{\alpha}\right\rangle=$ 0 . To evaluate the solvability condition at $\alpha=2$ we note the useful results

$$
\begin{aligned}
& \left\langle\mathrm{e}^{i k_{0} x}, \mathcal{L} u_{2}\right\rangle=0, \quad\left\langle\mathrm{e}^{i k_{0} x}, \beta_{2} w \otimes u_{1}^{2}\right\rangle=0, \quad\left\langle\mathrm{e}^{i k_{0} x}, \beta_{c} W^{x} \otimes \partial_{\chi} u_{1}\right\rangle=0, \\
& \left\langle\mathrm{e}^{i k_{0} x}, \gamma_{1} u_{1} \cos k_{f} x\right\rangle=\left\{\begin{array}{ll}
0 & n \neq 2 \\
\frac{\gamma_{1}}{2} A^{*} \mathrm{e}^{-2 i v x} & n=2
\end{array} .\right.
\end{aligned}
$$

Hence to avoid secular terms we must set $\gamma_{1}=0$ for the $2: 1$ resonance (with the solvability condition automatically guaranteed for all $\alpha \neq 2)$. We write $\gamma_{1}=\left(1-\delta_{n, 2}\right) \overline{\gamma_{1}}$. A particular solution of $u_{2}$ can be found by assuming that it is a linear combination of terms involving 
$\mathrm{e}^{i\left( \pm k_{f} \pm k_{0}\right)}$ and terms present in $u_{1}^{2}$. Substitution into (3.19) and balancing terms gives, for our balanced kernel $(\widehat{w}(0)=0)$,

$$
u_{2}=d_{0} A^{2} \mathrm{e}^{2 i k_{0} x}+\left(1-\delta_{n, 2}\right) \frac{\overline{\gamma_{1}}}{2}\left[d_{+} A \mathrm{e}^{i\left(k_{f}+k_{0}\right) x}+d_{-} A^{*} \mathrm{e}^{i\left(k_{f}-k_{0}\right) x}\right]+\text { c.c. },
$$

where

$$
d_{0}=\frac{\beta_{2} \widehat{w}\left(2 k_{0}\right)}{1-\beta_{c} \widehat{w}\left(2 k_{0}\right)}, \quad d_{ \pm}=\frac{1}{1-\beta_{c} \widehat{w}\left(k_{f} \pm k_{0}\right)}
$$

A similar analysis of the solvability condition at $\alpha=3$, and using the results in appendix A, gives the evolution of the amplitude $A$ as

$$
\begin{aligned}
\frac{\partial A}{\partial \tau}=\delta \widehat{w}\left(k_{0}\right) A & +\left[2 \beta_{2} \widehat{w}\left(k_{0}\right) d_{0}+3 \beta_{3} \widehat{w}\left(k_{0}\right)\right] A|A|^{2}-\frac{1}{2} \beta_{c} \widehat{w}^{\prime \prime}\left(k_{0}\right) \frac{\partial^{2} A}{\partial \chi^{2}} \\
& +\frac{\gamma_{2}}{2} A^{*} \mathrm{e}^{-2 i c \chi} \delta_{n, 2}+\left(1-\delta_{n, 2}\right)\left(\frac{\overline{\gamma_{1}}}{2}\right)^{2}\left[\left(d_{+}+d_{-}\right) A+A^{*} d_{-} \mathrm{e}^{-2 i c \chi} \delta_{n, 1}\right]
\end{aligned}
$$

If we now introduce the amplitude variable $a=\epsilon \mathrm{e}^{i c \chi} A$ then to leading order the solution for $u$ is of the form

$$
u-u_{0} \simeq a \mathrm{e}^{i k_{f} x / n}+\text { c.c. }
$$

After rescaling back to the original time and space variables the amplitude $a$ evolves according to

$$
\begin{aligned}
\beta_{c} \frac{\partial a}{\partial t}=\epsilon^{2} \delta a-\Phi|a|^{2} a+ & \frac{1}{2} \widehat{w}^{\prime \prime}\left(k_{0}\right)\left[\beta_{c}\left(v+i \partial_{x}\right)\right]^{2} a+\delta_{n, 2} \frac{\epsilon^{2} \gamma_{2}}{2} \beta_{c} a^{*} \\
& +\left(1-\delta_{n, 2}\right) \beta_{c}\left(\frac{\epsilon \overline{\gamma_{1}}}{2}\right)^{2}\left[\left(d_{+}+d_{-}\right) a+a^{*} d_{-} \delta_{n, 1}\right]
\end{aligned}
$$

where $\Phi=-3 \beta_{3}-2 \beta_{2} d_{0}$. Thus, from the solution form of (3.27), constant solutions of the amplitude equation (3.28) generate $n: 1$ resonant stationary stripe patterns. We next investigate the existence of such solutions for different values of $n$.

3.2.1. Existence of resonant stripe solutions. We consider the cases $n \neq 2$ and $n=2$ separately. For $n \neq 2$, equation (3.28) becomes,

$$
\beta_{c} \frac{\partial a}{\partial t}=\epsilon^{2} \delta a-\Phi|a|^{2} a+\frac{1}{2} \widehat{w}^{\prime \prime}\left(k_{0}\right)\left[\beta_{c}\left(v+i \partial_{x}\right)\right]^{2} a+\beta_{c}\left(\frac{\gamma}{2}\right)^{2}\left[\left(d_{+}+d_{-}\right) a+a^{*} d_{-} \delta_{n, 1}\right]
$$

where $\gamma=\epsilon \overline{\gamma_{1}}$. This has constant (resonant stripe) solutions of the form,

$$
a=\rho_{n} \mathrm{e}^{i \phi}, \quad \rho_{n}=\sqrt{\frac{4 \epsilon^{2} \delta+2 \widehat{w}^{\prime \prime}\left(k_{0}\right)\left(\beta_{c} v\right)^{2}+\beta_{c} \gamma^{2}\left[d_{+}+d_{-}\left(1+\delta_{n, 1}\right)\right]}{4 \Phi}} .
$$


For $n=1$ the constant argument $\phi \in\{0, \pi\}$, but for the higher order resonances the argument is undetermined by the amplitude equations to cubic order. In the case that $\Phi>0$ we find that the resonant stripe solutions exist for

$$
\gamma>\sqrt{\frac{-2 \widehat{w}^{\prime \prime}\left(k_{0}\right)\left(\beta_{c} v\right)^{2}-4 \epsilon^{2} \delta}{\beta_{c}\left[d_{+}+d_{-}\left(1+\delta_{n, 1}\right)\right]}} .
$$

For $n=2$, equation (3.28) becomes,

$$
\beta_{c} \frac{\partial a}{\partial t}=\epsilon^{2} \delta a-\Phi|a|^{2} a+\frac{1}{2} \widehat{w}^{\prime \prime}\left(k_{0}\right)\left[\beta_{c}\left(v+i \partial_{x}\right)\right]^{2} a+\frac{\gamma}{2} \beta_{c} a^{*},
$$

where $\gamma=\epsilon^{2} \gamma_{2}$. This has constant solutions of the form $a=\rho_{2} \mathrm{e}^{i \phi}$ where,

$$
\rho_{2}=\sqrt{\frac{2 \epsilon^{2} \delta+\widehat{w}^{\prime \prime}\left(k_{0}\right)\left(\beta_{c} v\right)^{2}+(-1)^{m} \gamma \beta_{c}}{2 \Phi}}, \quad \phi=\frac{m \pi}{2}, \quad m \in \mathbb{Z} .
$$

The solutions with $m$ odd are unstable so we do not consider these further. Assuming that $m$ is even and also that $\Phi>0$, the resonant stripe solutions exist for

$$
\gamma>-\widehat{w}^{\prime \prime}\left(k_{0}\right) \beta_{c} v^{2}-2 \epsilon^{2} \frac{\delta}{\beta_{c}} .
$$

The tongue shaped existence ranges for $n: 1$ resonant stripe patterns for $n=1, \ldots 4$ are shown in Figure 3. The parameter values are such that $\Phi>0$. We take $\epsilon^{2} \delta>0$ so that we are beyond the pattern forming instability. Notice that in this case the existence regions have finite width, even at $\gamma=0$ so the unforced system also supports bands of stripe solutions beyond the pattern forming instability. The 2:1 resonance tongue is noticeably wider than those for other resonances and we also note that narrow bands of the tongues for the $n: 1$ resonance patterns exists around $k_{f} / k_{0}=2$ for all values of $n \neq 2$. This is due to the fact that the tongue for $n=2$ has a different form to those for other values of $n$. For $n=2$, the forcing strength coefficient $\gamma$ appears linearly in the amplitude equation and therefore forcing has a stronger effect in this case than when $n \neq 2$ where $\gamma$ appears squared. This difference in the power to which $\gamma$ is raised in the amplitude equation occurs as a direct result of the forcing function $I(\mathbf{r}, t)$ being applied to a linear term in $u$ in (2.1). If for instance we were to add forcing via a cubic order term in $u$ we would expect to see a prominent 4:1 resonance. 


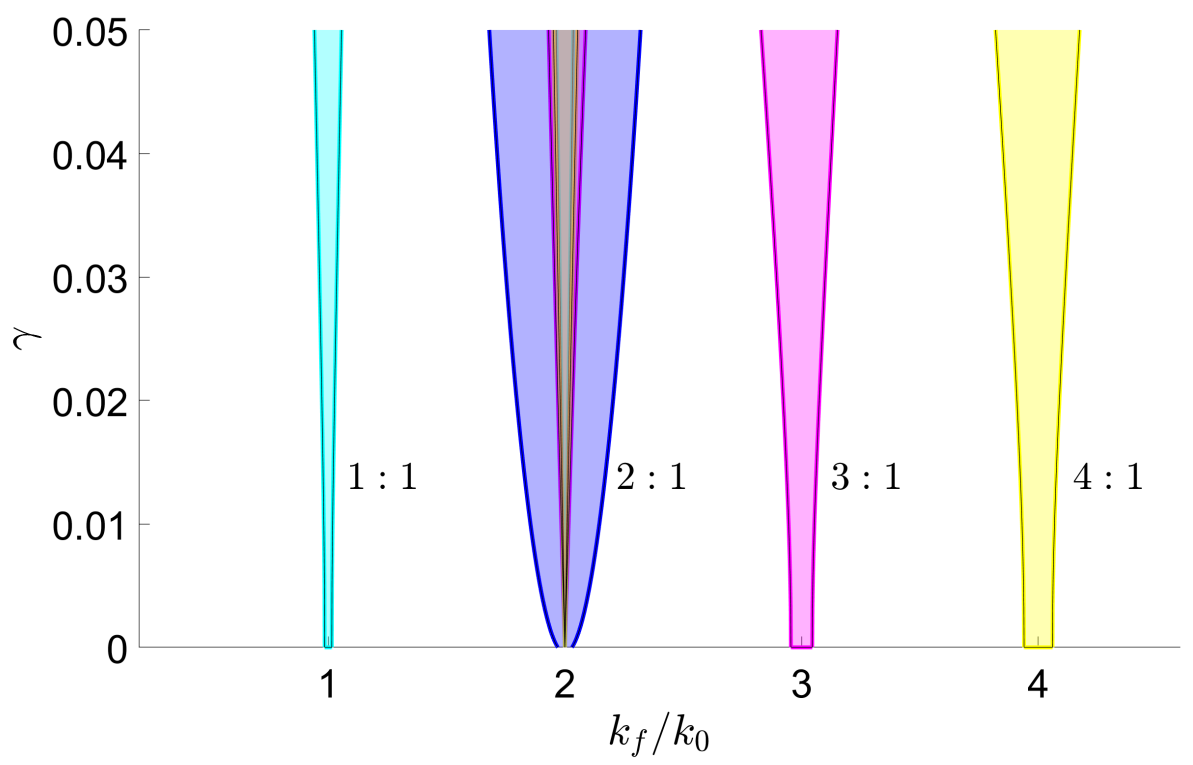

Figure 3: Existence tongues for resonant stripe patterns in a one-dimensional neural field model (without adaptation) with spatially periodic forcing. The kernel is chosen as in (2.4) with $\sigma=0.8$. Other parameters are $h=0$ and $\epsilon^{2} \delta=10^{-4}$. The tongue with a 2:1 resonance is dominant.

4. Weakly nonlinear analysis. We now carry out a weakly nonlinear analysis of the fully two-dimensional model including adaptation which allows us to derive amplitude equations for the emergent patterns in the neighbourhood of a Turing instability. The resulting four (complex valued) amplitude equations can be reduced to a four-dimensional system in two ways. We consider in section 4.1 the two-dimensional model without adaptation $(g=0)$ and use bifurcation theory to investigate the spatial patterns which are supported. We focus on discovering the parameter choices which give an orthogonal response to the spatially periodic forcing. In section 4.2 we make a reduction to one spatial dimension with adaptation and investigate the effects of forcing on travelling waves.

At the Turing instability all wavevectors $\mathbf{k}=\left(k_{x}, k_{y}\right)$ of magnitude $|\mathbf{k}|=k_{0}$ are excited. We investigate solutions which are locked to the forcing wavevector $\mathbf{k}_{\mathbf{f}}=\left(k_{f}, 0\right)$. Here, $n: 1$ resonant solutions have

$$
k_{x}=\frac{k_{f}}{n}+v_{1}=k_{0}-v_{2}
$$

(see Figure 4) where the mismatch parameters $v_{1}, v_{2}$ satisfy $\left|v_{1}+k_{f} / n\right| \leq k_{0}$ and equivalently $0 \leq v_{2} \leq 2 k_{0}$. The spatial structure of the two-dimensional patterns that form are, to leading order, a superposition of the modes $\exp \left(i k_{x} x \pm i k_{y} y\right)$, which can lead to rectangular (equal amplitude) and oblique (unequal amplitude) patterns. These are $n: 1$ resonant patterns that respond to the spatial forcing by locking the wavevector components in the forcing direction $k_{x}=k_{f} / n$ and creating a wavevector component in the orthogonal direction, $k_{y}$, to compensate for the unfavourable forcing wave number, so that $k_{y}=\sqrt{k_{0}^{2}-k_{x}^{2}}$ to achieve the 
total wavenumber $k_{0}$. Note that if we fix $v_{1}=0$ as in [20] then dependence of existence and stability of solutions patterns on the mismatch $v_{2}$ occurs through $k_{x}$ and $k_{y}$. While we will see that this suffices when considering patterns in two spatial dimensions, in section 4.2 we make a reduction to one spatial dimension by setting $k_{y}=0$. In this case we have $v_{2}=0$ so that $k_{x}=k_{0}$ and we must have $v_{1}=k_{0}-k_{f} / n \neq 0$ so that $k_{x}$ depends on the mismatch $v_{1}$. In order to enable this reduction, we retain the mismatch parameter $v_{1}$ in the computation of amplitude equations for emergent patterns in the fully two-dimensional model including adaptation.

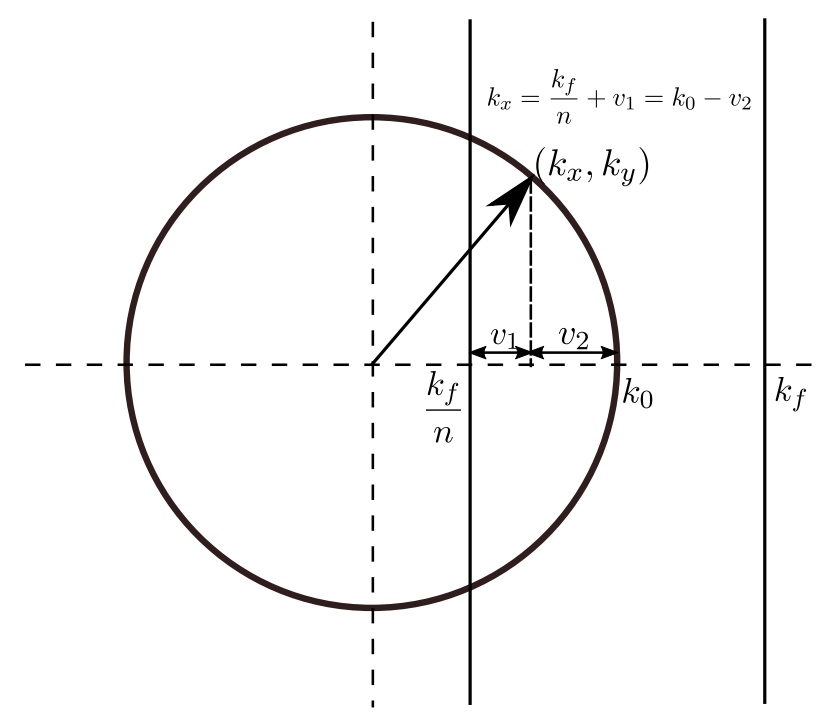

Figure 4: The circle indicates the ring of fastest growing wavenumbers with critical value $|\mathbf{k}|=k_{0}$, for $\mathbf{k}=\left(k_{x}, k_{y}\right)$. The forcing wavevector is $\mathbf{k}_{\mathbf{f}}=\left(k_{f}, 0\right)$. We take $k_{x}=k_{f} / n+v_{1}=$ $k_{0}-v_{2}$ for mismatch parameters $v_{1}$ and $v_{2}$, with $n \in \mathbb{Z}$. The wavevector component $k_{y}$ satisfies $k_{y}^{2}=k_{0}^{2}-k_{x}^{2}$ to achieve the total wavenumber $k_{0}$. The unforced system can support a spatially periodic Turing pattern with $|\mathbf{k}|=k_{0}$. With the introduction of forcing there are wide regions in parameter space that support a resonance with $n=2$ leading to the formation of rectangular and oblique solutions.

For the weakly nonlinear analysis we define new coordinates $\chi=\epsilon x, \Upsilon=\epsilon y, \tau=\epsilon^{2} t$ for a small parameter $\epsilon$ and consider power series expansions for $u$ and $\gamma$ as in (3.14)-(3.15) with as yet unknown functions $u_{\alpha}=u_{\alpha}(x, y, t, \chi, \Upsilon, \tau), \alpha=1,2,3, \ldots$ We again use the Taylor series expansion for the firing rate function $f(u)=f\left(u_{0}\right)+\beta_{1}\left(u-u_{0}\right)+\beta_{2}\left(u-u_{0}\right)^{2}+\beta_{3}\left(u-u_{0}\right)^{3}+\ldots$, where $\beta_{2}=f^{\prime \prime}\left(u_{0}\right) / 2, \beta_{3}=f^{\prime \prime \prime}\left(u_{0}\right) / 6$, and we treat $\beta_{1}$ as a bifurcation parameter and write $\beta_{1}=\beta_{c}+\epsilon^{2} \delta$ where now $\beta_{c}=f^{\prime}\left(u_{0}\right)$ subject to

$$
\beta_{c}= \begin{cases}\frac{1+g}{\widehat{w}\left(k_{0}\right)} & \text { at a static Turing bifurcation, } \\ \frac{\tau_{a}+1}{\tau_{a} \widehat{w}\left(k_{0}\right)} & \text { at a dynamic Turing instability }\end{cases}
$$


We must now also consider further Taylor expansions of the functions $u_{\alpha}$ to allow for the evaluation of the spatial and temporal convolutions in (3.12):

$$
\begin{gathered}
u_{\alpha}\left(x^{\prime}, y^{\prime}, t^{\prime}, \epsilon x^{\prime}, \epsilon y^{\prime}, \epsilon^{2} t^{\prime}\right)=u_{\alpha}\left(x^{\prime}, y^{\prime}, t^{\prime}, \chi+\epsilon\left(x^{\prime}-x\right), \Upsilon+\epsilon\left(y^{\prime}-y\right), \epsilon^{2} t^{\prime}\right) \\
\simeq u_{\alpha}\left(x^{\prime}, y^{\prime}, t^{\prime}, \chi, \Upsilon, \tau\right)+\epsilon\left(x^{\prime}-x\right) \frac{\partial}{\partial \chi} u_{\alpha}\left(x^{\prime}, y^{\prime}, t^{\prime}, \chi, \Upsilon, \tau\right)+\epsilon\left(y^{\prime}-y\right) \frac{\partial}{\partial \Upsilon} u_{\alpha}\left(x^{\prime}, y^{\prime}, t^{\prime}, \chi, \Upsilon, \tau\right) \\
+\frac{1}{2} \epsilon^{2}\left[\left(x^{\prime}-x\right)^{2} \frac{\partial^{2}}{\partial \chi^{2}} u_{\alpha}\left(x^{\prime}, y^{\prime}, t^{\prime}, \chi, \Upsilon, \tau\right)+2\left(x^{\prime}-x\right)\left(y^{\prime}-y\right) \frac{\partial^{2}}{\partial \chi \partial \Upsilon} u_{\alpha}\left(x^{\prime}, y^{\prime}, t^{\prime}, \chi, \Upsilon, \tau\right)\right. \\
\left.+\left(y^{\prime}-y\right)^{2} \frac{\partial^{2}}{\partial \Upsilon^{2}} u_{\alpha}\left(x^{\prime}, y^{\prime}, t^{\prime}, \chi, \Upsilon, \tau\right)\right]+O\left(\epsilon^{3}\right), \\
u_{\alpha}\left(x^{\prime}, y^{\prime}, t^{\prime}, \epsilon x^{\prime}, \epsilon y^{\prime}, \epsilon^{2} t^{\prime}\right)=u_{\alpha}\left(x^{\prime}, y^{\prime}, t^{\prime}, \epsilon x^{\prime}, \epsilon y^{\prime}, \tau+\epsilon^{2}\left(t^{\prime}-t\right)\right) \\
\simeq u_{\alpha}\left(x^{\prime}, y^{\prime}, t^{\prime}, \chi, \Upsilon, \tau\right)+\epsilon^{2}\left(t^{\prime}-t\right) \frac{\partial}{\partial \tau} u_{\alpha}\left(x^{\prime}, y^{\prime}, t^{\prime}, \chi, \Upsilon, \tau\right)+O\left(\epsilon^{4}\right) .
\end{gathered}
$$

Balancing the $O(1)$ terms in (3.12) fixes the steady state $u_{0}=0$ since we choose a balanced kernel as in (2.4) with $A=\sigma^{-2}$. Balancing terms at higher powers of $\epsilon$ in (3.12) yields a hierarchy of equations as

$$
\begin{aligned}
\mathcal{L}_{g} u_{1}= & 0 \\
\mathcal{L}_{g} u_{2}= & -M_{0}\left(\beta_{2} u_{1}^{2}\right)-M_{1}\left(\beta_{c} u_{1}\right)-\gamma_{1} u_{1} \cos \left(k_{f} x\right), \\
\mathcal{L}_{g} u_{3}= & \frac{\partial u_{1}}{\partial \tau}-M_{0}\left(\delta u_{1}+2 \beta_{2} u_{1} u_{2}+\beta_{3} u_{1}^{3}\right)-M_{1}\left(\beta_{c} u_{2}+\beta_{2} u_{1}^{2}\right) \\
& \quad-M_{2}\left(\beta_{c} u_{1}\right)+N_{1}\left(g u_{1}\right)-\left(\gamma_{1} u_{2}+\gamma_{2} u_{1}\right) \cos \left(k_{f} x\right),
\end{aligned}
$$

where we define the linear operators $\mathcal{L}_{g}=-\frac{\partial}{\partial t}-1+\beta_{c} w \otimes-g \eta *, M_{0}=w \otimes, M_{1}=W^{x} \otimes$ $\partial_{\chi}+W^{y} \otimes \partial_{\Upsilon}, M_{2}=\frac{1}{2}\left[W^{x x} \otimes \partial_{\chi \chi}+2 W^{x y} \otimes \partial_{\chi \Upsilon}+W^{y y} \otimes \partial_{\Upsilon \Upsilon}\right]$ and $N_{1}=\eta^{t} * \partial_{\tau}$. Here we have introduced new spatial kernels $W^{x}(\mathbf{r})=-w(|\mathbf{r}|) x$ and $W^{x y}(\mathbf{r})=w(|\mathbf{r}|) x y$ analogously to the scalar case in section 3.2. We also introduce the new temporal kernel $\eta^{t}(t)=-t \eta(t)$. The null space of the linear operator $\mathcal{L}_{g}$ is spanned by $\left\{\mathrm{e}^{ \pm i\left(k_{x} x \pm k_{y} y \pm \omega_{c} t\right)}\right\}$ where $k_{x}^{2}+k_{y}^{2}=k_{0}^{2}$, $\omega_{c}=\sqrt{\tau_{a} g-1} / \tau_{a}$, and therefore (4.2) has solution

$$
\begin{aligned}
u_{1}(x, y, t, \chi, \Upsilon, \tau)= & A_{1}(\chi, \Upsilon, \tau) \mathrm{e}^{i\left(k_{x} x+k_{y} y+\omega_{c} t\right)}+A_{2}(\chi, \Upsilon, \tau) \mathrm{e}^{i\left(k_{x} x-k_{y} y+\omega_{c} t\right)} \\
& +A_{3}(\chi, \Upsilon, \tau) \mathrm{e}^{i\left(k_{x} x+k_{y} y-\omega_{c} t\right)}+A_{4}(\chi, \Upsilon, \tau) \mathrm{e}^{i\left(k_{x} x-k_{y} y-\omega_{c} t\right)}+\text { c.c. }
\end{aligned}
$$

Using the Fredholm alternative we find a particular solution to (4.3) and use a solvability condition for (4.4) to derive amplitude equations for the evolution of the complex amplitudes $A_{j}(\chi, \Upsilon, \tau), j=1,2,3,4$. Details of these calculations can be found in Appendix B and the resulting amplitude equations, rescaled back to the original time and space variables are

$$
\begin{aligned}
\left(1+g \widetilde{\eta}^{\prime}\left(i \omega_{c}\right)\right) \frac{\partial a_{1}}{\partial t}= & -\widehat{w}\left(k_{0}\right)\left(\left(\Phi_{1}\left|a_{1}\right|^{2}+\Phi_{2}\left|a_{2}\right|^{2}+\Phi_{3}\left|a_{3}\right|^{2}+\Phi_{4}\left|a_{4}\right|^{2}\right) a_{1}+\Phi_{5} a_{2} a_{3} a_{4}^{*}\right) \\
+ & \widehat{w}\left(k_{0}\right) \epsilon^{2} \delta a_{1}+\frac{\beta_{c}}{2} \widehat{w}^{\prime \prime}\left(k_{0}\right)\left(\left(i \partial_{x}+v_{1}\right)^{2}-\partial_{y y}\right) a_{1}+\frac{\epsilon^{2} \gamma_{2}}{2} a_{4}^{*} \delta_{n, 2} \\
& +\left(\frac{\epsilon \bar{\gamma}_{1}}{2}\right)^{2}\left(1-\delta_{n, 2}\right)\left[\left(\zeta_{+}+\zeta_{-}\right) a_{1}+\zeta_{-} a_{4}^{*} \delta_{n, 1}\right]
\end{aligned}
$$




$$
\begin{aligned}
\left(1+g \widetilde{\eta}^{\prime}\left(i \omega_{c}\right)\right) \frac{\partial a_{2}}{\partial t}= & -\widehat{w}\left(k_{0}\right)\left(\left(\Phi_{2}\left|a_{1}\right|^{2}+\Phi_{1}\left|a_{2}\right|^{2}+\Phi_{4}\left|a_{3}\right|^{2}+\Phi_{3}\left|a_{4}\right|^{2}\right) a_{2}+\Phi_{5} a_{1} a_{4} a_{3}^{*}\right) \\
& +\widehat{w}\left(k_{0}\right) \epsilon^{2} \delta a_{2}+\frac{\beta_{c}}{2} \widehat{w}^{\prime \prime}\left(k_{0}\right)\left(\left(i \partial_{x}+v_{1}\right)^{2}-\partial_{y y}\right) a_{2}+\frac{\epsilon^{2} \gamma_{2}}{2} a_{3}^{*} \delta_{n, 2} \\
& +\left(\frac{\epsilon \bar{\gamma}_{1}}{2}\right)^{2}\left(1-\delta_{n, 2}\right)\left[\left(\zeta_{+}+\zeta_{-}\right) a_{2}+\zeta_{-} a_{3}^{*} \delta_{n, 1}\right],
\end{aligned}
$$

$$
\left(1+g \widetilde{\eta}^{\prime}\left(-i \omega_{c}\right)\right) \frac{\partial a_{3}}{\partial t}=-\widehat{w}\left(k_{0}\right)\left(\left(\Phi_{3}^{*}\left|a_{1}\right|^{2}+\Phi_{4}^{*}\left|a_{2}\right|^{2}+\Phi_{1}^{*}\left|a_{3}\right|^{2}+\Phi_{2}^{*}\left|a_{4}\right|^{2}\right) a_{3}+\Phi_{5}^{*} a_{1} a_{4} a_{2}^{*}\right)
$$

$$
+\widehat{w}\left(k_{0}\right) \epsilon^{2} \delta a_{3}+\frac{\beta_{c}}{2} \widehat{w}^{\prime \prime}\left(k_{0}\right)\left(\left(i \partial_{x}+v_{1}\right)^{2}-\partial_{y y}\right) a_{3}+\frac{\epsilon^{2} \gamma_{2}}{2} a_{2}^{*} \delta_{n, 2}
$$

$$
+\left(\frac{\epsilon \bar{\gamma}_{1}}{2}\right)^{2}\left(1-\delta_{n, 2}\right)\left[\left(\zeta_{+}^{*}+\zeta_{-}^{*}\right) a_{3}+\zeta_{-}^{*} a_{2}^{*} \delta_{n, 1}\right]
$$

$$
\begin{aligned}
\left(1+g \widetilde{\eta}^{\prime}\left(-i \omega_{c}\right)\right) \frac{\partial a_{4}}{\partial t}= & -\widehat{w}\left(k_{0}\right)\left(\left(\Phi_{4}^{*}\left|a_{1}\right|^{2}+\Phi_{3}^{*}\left|a_{2}\right|^{2}+\Phi_{2}^{*}\left|a_{3}\right|^{2}+\Phi_{1}^{*}\left|a_{4}\right|^{2}\right) a_{4}+\Phi_{5}^{*} a_{2} a_{3} a_{1}^{*}\right) \\
+ & \widehat{w}\left(k_{0}\right) \epsilon^{2} \delta a_{4}+\frac{\beta_{c}}{2} \widehat{w}^{\prime \prime}\left(k_{0}\right)\left(\left(i \partial_{x}+v_{1}\right)^{2}-\partial_{y y}\right) a_{4}+\frac{\epsilon^{2} \gamma_{2}}{2} a_{1}^{*} \delta_{n, 2} \\
& +\left(\frac{\epsilon \bar{\gamma}_{1}}{2}\right)^{2}\left(1-\delta_{n, 2}\right)\left[\left(\zeta_{+}^{*}+\zeta_{-}^{*}\right) a_{4}+\zeta_{-}^{*} a_{1}^{*} \delta_{n, 1}\right]
\end{aligned}
$$

where $\Phi_{1}, \ldots, \Phi_{5}$ are as in (B.25). These four complex-valued coupled nonlinear ODEs describe the evolution of the amplitudes $a_{j}(x, y, t), j=1,2,3,4$ in the solution $u$ of (3.12) which to leading order is given by

$$
u(x, y, t)=\mathrm{e}^{i k_{f} x / n}\left(a_{1} \mathrm{e}^{i\left(k_{y} y+\omega t\right)}+a_{2} \mathrm{e}^{i\left(-k_{y} y+\omega t\right)}+a_{3} \mathrm{e}^{i\left(k_{y} y-\omega t\right)}+a_{4} \mathrm{e}^{i\left(-k_{y} y-\omega t\right)}\right)+\text { c.c. }
$$

where $\omega=\omega_{c}+\xi$ is the (temporal) frequency of the solution away from bifurcation and $\xi$ is an order $\epsilon^{2}$ temporal frequency detuning parameter which does not appear in the amplitude equations (see Appendix B). Constant solutions of the amplitude equations (4.6)-(4.9) for a given value of $n$ correspond to $n: 1$ resonant patterns which exist beyond the Turing instability. We have a particular interest in resonant patterns under the one-dimensional forcing in the $x$ direction which have a wavevector component in the orthogonal direction. This orthogonal response is seen in the Swift-Hohenberg equation for the 2:1 resonance [20] where stable rectangles and oblique patterns are observed. We therefore also choose to focus on the 2:1 resonance. In section 4.1 we consider two-dimensional spatial patterns near a static instability in the model without adaptation $(g=0)$, while in section 4.2 we consider the model with adaptation where the unforced system supports travelling waves beyond the dynamic Turing instability. (Note that for the study of dynamic patterns we make a reduction to one spatial dimension to simplify calculations.) 
4.1. Spatial patterns without adaptation. First consider the $2: 1$ resonance in the case where there is no adaptation so that there is a static Turing instability at $\beta_{c}=1 / \widehat{w}\left(k_{0}\right)$. We set $g=0$ and $\omega_{c}=0$ in (4.6)-(4.9) and also we let $a_{2}=a_{3}=0$ as these terms are no longer needed in $u_{1}$ given by (4.5) when $\omega_{c}=0$ (since the null space of $\mathcal{L}$ is spanned by the terms with coefficients $A_{1}$ and $A_{4}$ in (4.5)). We also choose to set $v_{1}=0$ so that $k_{x}=k_{f} / 2=k_{0}-v_{2}$ and dependence on the mismatch between $k_{f}$ and $k_{0}$ enters the amplitude equations through $k_{x}$ and $k_{y}$, noting that $\Phi_{4}$ depends on these parameters. We then have the following amplitude equations for $a_{1}=a$ and $a_{4}=b$ :

$$
\begin{aligned}
& \beta_{c} \frac{\partial a}{\partial t}=\epsilon^{2} \delta a-\Phi_{1}|a|^{2} a-\Phi_{4}|b|^{2} a-\frac{\beta_{c}^{2}}{2} \widehat{w}^{\prime \prime}\left(k_{0}\right)\left(\partial_{x x}+\partial_{y y}\right) a+\frac{\gamma \beta_{c}}{2} b^{*}, \\
& \beta_{c} \frac{\partial b}{\partial t}=\epsilon^{2} \delta b-\Phi_{1}|b|^{2} b-\Phi_{4}|a|^{2} b-\frac{\beta_{c}^{2}}{2} \widehat{w}^{\prime \prime}\left(k_{0}\right)\left(\partial_{x x}+\partial_{y y}\right) b+\frac{\gamma \beta_{c}}{2} a^{*},
\end{aligned}
$$

where $\gamma=\epsilon^{2} \gamma_{2}$ and we note that the coefficients $\Phi_{1}$ and $\Phi_{4}$ are real. These equations have a similar structure to those for two-dimensional patterns in the spatially forced SwiftHohenberg equation [20]. We now look for spatially homogeneous solutions of (4.11)-(4.12). Writing $a=\rho_{a} \mathrm{e}^{i \phi_{a}}$ and $b=\rho_{b} \mathrm{e}^{i \phi_{b}}$ we find that the phases and amplitudes satisfy

$$
\begin{aligned}
\beta_{c} \frac{\partial \rho_{a}}{\partial t} & =\epsilon^{2} \delta \rho_{a}-\Phi_{1} \rho_{a}^{3}-\Phi_{4} \rho_{a} \rho_{b}^{2}+\frac{\gamma \beta_{c}}{2} \rho_{b} \cos (\psi), \\
\beta_{c} \frac{\partial \rho_{b}}{\partial t} & =\epsilon^{2} \delta \rho_{b}-\Phi_{1} \rho_{b}^{3}-\Phi_{4} \rho_{a}^{2} \rho_{b}+\frac{\gamma \beta_{c}}{2} \rho_{a} \cos (\psi), \\
\frac{\partial \psi}{\partial t} & =-\frac{\gamma}{2}\left(\frac{\rho_{b}}{\rho_{a}}+\frac{\rho_{a}}{\rho_{b}}\right) \sin (\psi), \\
\frac{\partial \theta}{\partial t} & =-\frac{\gamma}{2}\left(\frac{\rho_{b}}{\rho_{a}}-\frac{\rho_{a}}{\rho_{b}}\right) \sin (\psi),
\end{aligned}
$$

where $\psi=\phi_{a}+\phi_{b}$ and $\theta=\phi_{a}-\phi_{b}$. Notice that $\theta$ is determined once $\rho_{a}, \rho_{b}$ and $\psi$ are known. Looking for constant solutions we find that $\psi=m \pi, m=0,1$, however such solutions with $\psi=\pi$ can be shown to be unstable and therefore we do not consider these further. With $\psi=0$, we see from (4.16) that phases $\phi_{a}$ and $\phi_{b}=-\phi_{a}$ are constant and that constant non-zero amplitudes $\rho_{a}, \rho_{b}$ satisfy

$$
\epsilon^{2} \delta \rho_{a}-\Phi_{1} \rho_{a}^{3}-\Phi_{4} \rho_{a} \rho_{b}^{2}+\frac{\gamma \beta_{c}}{2} \rho_{b}=0, \quad \epsilon^{2} \delta \rho_{b}-\Phi_{1} \rho_{b}^{3}-\Phi_{4} \rho_{a}^{2} \rho_{b}+\frac{\gamma \beta_{c}}{2} \rho_{a}=0 .
$$

Equations (4.17) admit the solution $\rho_{a}=\rho_{b}=\rho_{0}$ where

$$
\rho_{0}=\sqrt{\frac{2 \epsilon^{2} \delta+\gamma \beta_{c}}{2\left(\Phi_{1}+\Phi_{4}\right)}} .
$$

These are constant rectangular patterns

$$
\begin{aligned}
u(x, y, t) & =\rho_{0} \mathrm{e}^{i k_{f} x / 2}\left(\mathrm{e}^{i\left(k_{y} y+\phi_{a}\right)}+\mathrm{e}^{-i\left(k_{y} y+\phi_{a}\right)}\right)+\text { c.c. } \\
& =4 \rho_{0} \cos \left(k_{f} x / 2\right) \cos \left(k_{y} y+\phi_{a}\right)
\end{aligned}
$$


where $k_{y}=\sqrt{k_{0}^{2}-k_{x}^{2}}, \quad k_{x}=k_{f} / 2=k_{0}-v_{2}$. The undetermined phase $\phi_{a}$ arises due to the continuous translational symmetry in the $y$-direction which is not broken by the forcing. These solutions exist for $0<v_{2}<2 k_{0}$ (to ensure that $k_{y} \in \mathbb{R}$ ) and where also $2 \epsilon^{2} \delta+\gamma \beta_{c}$ and $\Phi_{1}+\Phi_{4}$ have the same sign, noting that $\Phi_{4}=\Phi_{4}\left(v_{2}\right)$.

Equations (4.17) also admit the constant solution $\rho_{a}=\rho_{ \pm}, \rho_{b}=\rho_{\mp}$ where

$$
\rho_{ \pm}^{2}=\frac{\epsilon^{2} \delta}{2 \Phi_{1}} \pm \sqrt{\left(\frac{\epsilon^{2} \delta}{2 \Phi_{1}}\right)^{2}-\left(\frac{\gamma \beta_{c}}{2\left(\Phi_{1}-\Phi_{4}\right)}\right)^{2}} .
$$

These are constant oblique patterns

$$
\begin{aligned}
u(x, y, t) & =\mathrm{e}^{i k_{f} x / 2}\left(\rho_{ \pm} \mathrm{e}^{i\left(k_{y} y+\phi_{a}\right)}+\rho_{\mp} \mathrm{e}^{-i\left(k_{y} y+\phi_{a}\right)}\right)+\text { c.c. } \\
& =2 \rho_{ \pm} \cos \left(k_{f} x / 2+k_{y} y+\phi_{a}\right)+2 \rho_{\mp} \cos \left(k_{f} x / 2-k_{y} y-\phi_{a}\right),
\end{aligned}
$$

where $\phi_{a}$ is again undetermined and $k_{y}=\sqrt{k_{0}^{2}-k_{x}^{2}}, \quad k_{x}=k_{f} / 2=k_{0}-v_{2}$. These solutions exist for $0<v_{2}<2 k_{0}$ (to ensure that $k_{y} \in \mathbb{R}$ ) and where also

$$
\frac{\epsilon^{2} \delta}{2 \Phi_{1}}>0 \quad \text { and } \quad|\gamma|<\frac{\epsilon^{2} \delta}{\beta_{c} \Phi_{1}}\left|\Phi_{1}-\Phi_{4}\right| .
$$

The values of $v_{2}$ and $\gamma$ for which resonant rectangle and oblique patterns exist depend on the values of $\sigma$ (the spatial scale of interaction) and $h$ (the firing rate threshold). The existence regions for a range of values of $h$ for $\sigma=0.5$ are illustrated in Figure 5. Regions where rectangle patterns exist are shaded blue, while red shading indicates existence of oblique patterns under the additional assumption that $\epsilon^{2} \delta / \Phi_{1}>0$. For $h=0$ we observe similar existence regions for these patterned states as observed in [20] for the Swift-Hohenberg equation under periodic spatial forcing. For nonzero choices of $h$ we observe more complex existence regions. We note that the existence regions for $-h$ are identical to those for $h$. This is due to the fact that $f^{\prime}\left(u_{0}\right)$ is an even function of $h$. The values of $\beta_{2}$ and $\beta_{3}$ depend on $\mu$ where $\mu$ is fixed once $h$ and $\sigma$ are specified. Since for given a given value of $h, \mu$ satisfies $\beta_{c}=1 / \widehat{w}\left(k_{0}\right)=f^{\prime}\left(u_{0}\right)$, then $-h$ gives the same values of $\mu$ as $h$.

We can also consider the linear stability of the two-dimensional constant resonance patterns to uniform perturbations. Making perturbations $\Delta \rho_{a}$ and $\Delta \rho_{b}$ to the constant solution $\rho_{a}, \rho_{b}$ and linearising we find that the perturbations satisfy

$$
\frac{\partial}{\partial t}\left(\begin{array}{c}
\Delta \rho_{a} \\
\Delta \rho_{b}
\end{array}\right)=\frac{1}{\beta_{c}}\left(\begin{array}{cc}
\epsilon^{2} \delta-3 \Phi_{1} \rho_{a}^{2}-\Phi_{4} \rho_{b}^{2} & -2 \Phi_{4} \rho_{a} \rho_{b}+\frac{\gamma \beta_{c}}{2} \\
-2 \Phi_{4} \rho_{a} \rho_{b}+\frac{\gamma \beta_{c}}{2} & \epsilon^{2} \delta-3 \Phi_{1} \rho_{b}^{2}-\Phi_{4} \rho_{a}^{2}
\end{array}\right)\left(\begin{array}{c}
\Delta \rho_{a} \\
\Delta \rho_{b}
\end{array}\right) .
$$

The Jacobian, $J$, in (4.20) has eigenvalues

$$
\lambda_{ \pm}=\frac{\operatorname{Tr}(J)}{2} \pm \frac{1}{2} \sqrt{(\operatorname{Tr}(J))^{2}-4 \operatorname{Det}(J)}
$$

The zero state $\left(\rho_{a}=\rho_{b}=0\right)$ has eigenvalues $\left(\epsilon^{2} \delta / \beta_{c}\right) \pm \gamma / 2$ and is therefore stable for $2 \epsilon^{2} \delta \pm \gamma \beta_{c}<0$ since $\beta_{c}>0$. Rectangular patterns have $\rho_{a}=\rho_{b}=\rho_{0}$ and eigenvalues

$$
\lambda_{+}=-2\left(\frac{\epsilon^{2} \delta}{\beta_{c}}+\frac{\gamma}{2}\right), \quad \lambda_{-}=\frac{-2\left(\Phi_{1}-\Phi_{4}\right) \epsilon^{2} \delta-2 \Phi_{1} \gamma \beta_{c}}{\beta_{c}\left(\Phi_{1}+\Phi_{4}\right)} .
$$




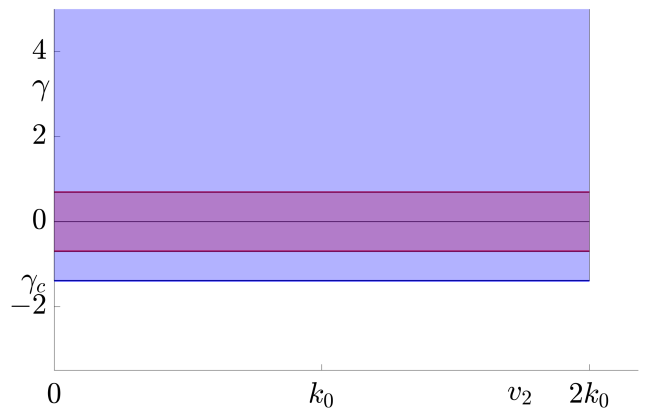

(a) $h=0$

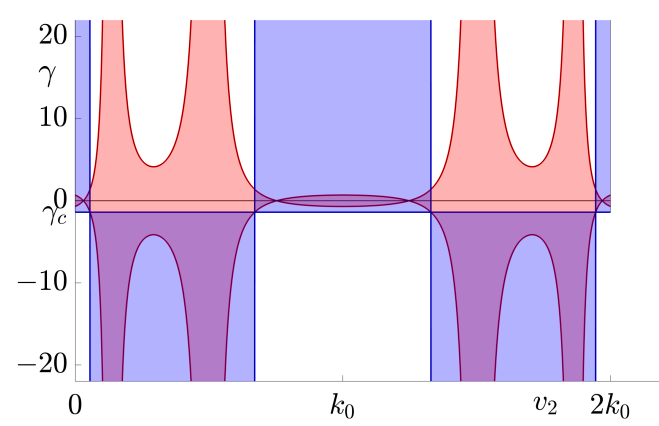

(c) $|h|=0.3$

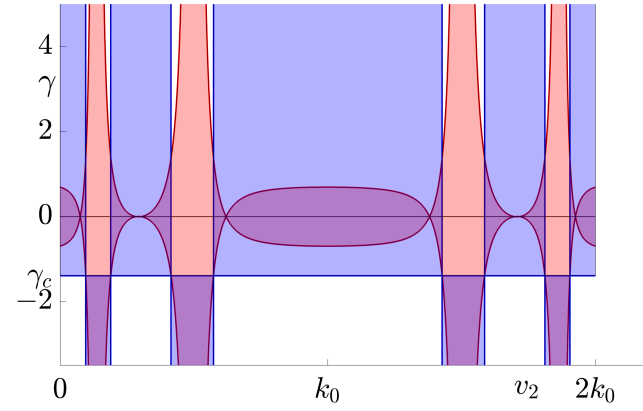

(b) $|h|=0.15$

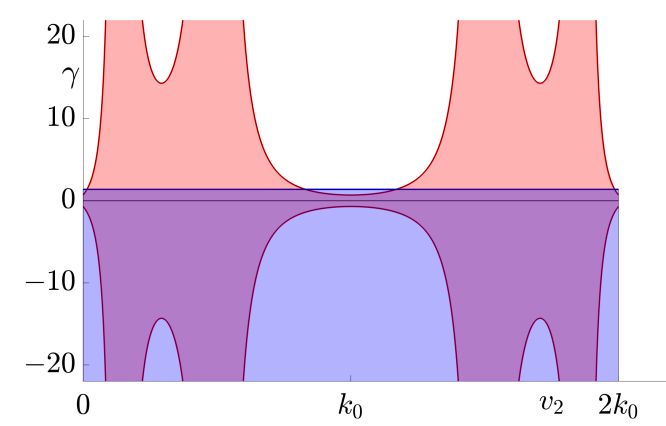

(d) $|h|=0.5$

Figure 5: Existence regions for patterned states in a two-dimensional neural field model with spatially periodic forcing (and without adaptation). Blue shaded regions indicate where stationary rectangle patterns exist and red shading indicates existence of oblique patterns. The kernel is chosen as in (2.4) with $\sigma=0.5$ and the firing rate is given by (2.5) with (a) $h=0$, (b) $|h|=0.15$, (c) $|h|=0.3$, (d) $|h|=0.5$. Other parameters are $\epsilon^{2} \delta=0.3$ for (a)-(c) and $\epsilon^{2} \delta=-0.3$ for $(\mathrm{d})$. Note that existence of oblique patterns also requires that $\epsilon^{2} \delta / \Phi_{1}>0$. Here $\gamma_{c}=-2 \epsilon^{2} \delta / \beta_{c}$.

Therefore rectangles are stable when $2 \epsilon^{2} \delta+\gamma \beta_{c}>0$ (and we need $\Phi_{1}+\Phi_{4}>0$ so that the solutions exist here) and also $\left(\Phi_{1}-\Phi_{4}\right) \epsilon^{2} \delta+\Phi_{1} \gamma \beta_{c}>0$. For oblique patterns, where $\rho_{a} \neq \rho_{b}$, we note from (4.17) that the constant solutions satisfy

$$
\epsilon^{2} \delta=\Phi_{1}\left(\rho_{a}^{2}+\rho_{b}^{2}\right) \quad \text { and } \quad \gamma \beta_{c}=-2 \rho_{a} \rho_{b}\left(\Phi_{1}-\Phi_{4}\right),
$$

and therefore the Jacobian matrix $J$ in (4.20) has

$$
\operatorname{Tr}(J)=-\left(\Phi_{1}+\Phi_{4}\right) \frac{\epsilon^{2} \delta}{\beta_{c} \Phi_{1}}, \quad \operatorname{Det}(J)=-2\left(\frac{\epsilon^{2} \delta}{\beta_{c} \Phi_{1}}\right)^{2} \Phi_{1}\left(\Phi_{1}-\Phi_{4}\right)+\left(\frac{3 \Phi_{1}-\Phi_{4}}{\Phi_{1}-\Phi_{4}}\right)^{2}\left(\frac{\gamma}{2}\right)^{2} .
$$

546 The oblique patterns are stable when $\operatorname{Tr}(J)<0$ and $\operatorname{Det}(J)>0$. The first of these conditions is satisfied when the patterns exist and $\Phi_{1}+\Phi_{4}>0$. Note then that all stable constant 
resonant two-dimensional patterns exist within the upper blue shaded regions in Figure 5a5 d. Stability regions in the $\left(v_{2}, \gamma\right)$ plane are indicated for rectangle and oblique patterns in Figure 6 for $\epsilon^{2} \delta=0.3$ and $|h|=0.15$. Stability results for $|h|=0.15$ are illustrated in the bifurcation diagrams in Figure 7 . There is a change in stability between rectangles and obliques at $\gamma=\gamma_{c}=\left(\Phi_{4}-\Phi_{1}\right) \epsilon^{2} \delta /\left(\Phi_{1} \beta_{c}\right)$ for fixed $\epsilon^{2} \delta$ or at $\left(\epsilon^{2} \delta\right)_{c}=\gamma \Phi_{1} \beta_{c} /\left(\Phi_{4}-\Phi_{1}\right)$ for fixed $\gamma$. The stable two-dimensional leading order pattern for values of $v_{2}$ increasing from 0 to $k_{0}$ (corresponding to $k_{x}$ decreasing from $k_{0}$ to 0 ) and a range of values of forcing strength $\gamma$ are shown in Figure 8. Here we choose $h=0$ so that stable two-dimensional leading order patterns exist for all values of $v_{2}$. As $v_{2}$ is increased from 0 to $k_{0}$ the pattern changes from vertical stripes to rectangles (when $\gamma>\gamma_{c}$ ) or oblique patterns (when $\gamma<\gamma_{c}$ ) to horizontal stripes which are orthogonal to the forcing. At $v_{2}=k_{0} / 4$ the rectangular patterns are square and the oblique patterns are precisely diagonal. Direct numerical simulations confirm that using the mismatch parameter $v_{2}$ to control the forcing can indeed lead to stripe patterns along the $x$-direction changing to stripe patterns along the $y$-direction. Thus, a simple neural field model can support an orthogonal response to patterned input.

The two-dimensional resonant patterns exist and are stable for a range of values of the detuning $v_{2}$ and these lie in 1,3 or 5 bands whose widths depend on the value of the firing rate threshold $h$. The width of these bands does not depend on $\gamma$ and hence the resonant patterns exist even in the limit of weak forcing $\gamma \rightarrow 0$. We also note in particular that a band of stable resonant orthogonal response patterns exists around $v_{2}=k_{0}$ for all $|h|<h_{c}$ where $h_{c} \approx 0.4196$ for $\sigma=0.5$.

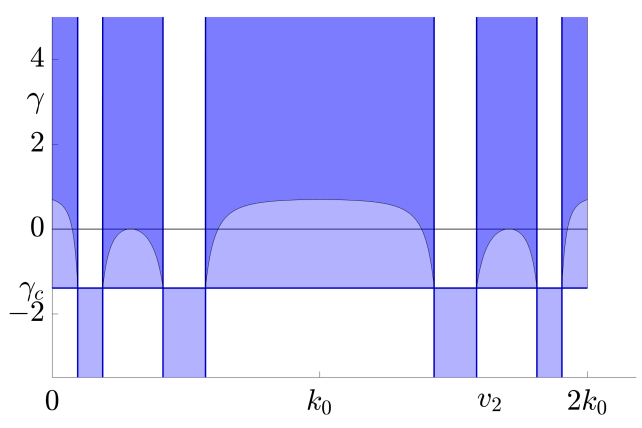

(a) Rectangles

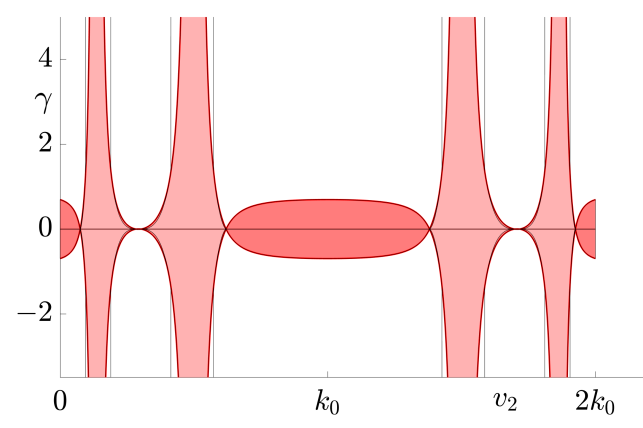

(b) Obliques

Figure 6: Stability tongues for constant two-dimensional 2:1 resonant solution patterns for the forced neural field equation (3.12) with no adaptation $(g=0)$. The left (right) diagram show the existence and stability tongues for rectangles (obliques). Darker shading indicates where the pattern is stable. Here $\sigma=0.5,|h|=0.15, \epsilon^{2} \delta=0.3$ and $\gamma_{c}=-2 \epsilon^{2} \delta / \beta_{c}$.

4.2. Waves with adaptation. We now consider the $2: 1$ resonance patterns that exist in the model with adaptation $((3.12)$ with $g \neq 0)$. Here, beyond the dynamic Turing instability at $\beta_{c}=\left(\tau_{a}+1\right) /\left(\tau_{a} \widehat{w}\left(k_{0}\right)\right)$, the unforced system $(\gamma=0)$ supports travelling waves. Due to the high dimension of the system of amplitude equations for the two-dimensional model with 

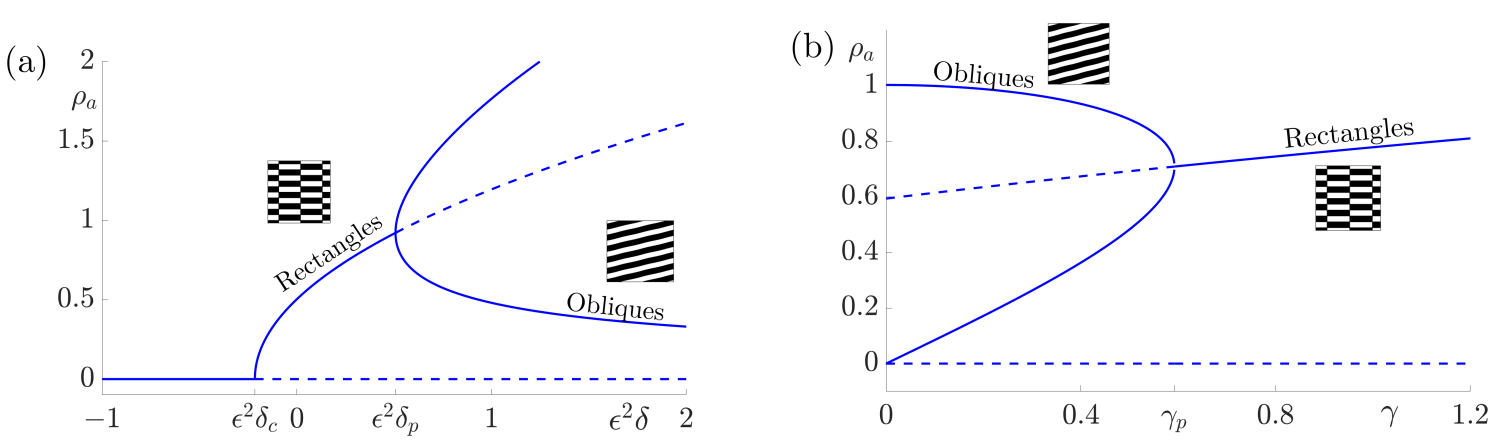

Figure 7: Bifurcation diagrams for constant two-dimensional pattern solutions for the forced neural field equation (3.12) with no adaptation $(g=0)$. Solid lines indicate stable states while dotted lines indicate unstable solutions. In both diagrams parameter values are $\sigma=0.5$, $|h|=0.15$ and $v_{2}=0.75 k_{0}$. In diagram (a) we fix forcing strength $\gamma=1$ and range over values of $\epsilon^{2} \delta$. Here the Turing bifurcation occurs at $\epsilon^{2} \delta_{c}=-\gamma \beta_{c} / 2$ and the bifurcation of rectangles to stable obliques occurs at $\epsilon^{2} \delta_{p}=\gamma \beta_{c} \Phi_{1} /\left(\Phi_{4}-\Phi_{1}\right)$. In diagram (b) we hold the distance from Turing instability, $\epsilon^{2} \delta=0.3$, and range over values of $\gamma$ with the bifurcation between patterned states at $\gamma_{p}=\left(\Phi_{4}-\Phi_{1}\right) \epsilon^{2} \delta /\left(\beta_{c} \Phi_{1}\right)$.

adaptation (4.6)-(4.9), to make analytical progress in establishing existence and stability of resonant dynamical patterns under one-dimensional spatial forcing, we reduce to one spatial dimension by taking $k_{y}=0$ and $v_{2}=0$ in (4.6)-(4.9) so that $k_{x}=k_{0}=k_{f} / n+v_{1}$. We continue to focus on the $2: 1$ resonance so we take $n=2$. We also let $a_{2}=a_{3}=0$ as these terms are no longer needed in $u_{1}$ given by (4.5) when $k_{y}=0$ (since the null space of $\mathcal{L}_{g}$ is spanned by the terms with coefficients $A_{1}$ and $A_{4}$ in (4.5)). We also now use the kernel (2.4) with $A=\sigma^{-1}$ which is balanced in one spatial dimension and has Fourier transform (3.21). We then have the following amplitude equations for $a_{1}=a$ and $a_{4}=b$ :

$$
\begin{gathered}
\left(1+g \widetilde{\eta}^{\prime}\left(i \omega_{c}\right)\right) \frac{\partial a}{\partial t}=\Lambda a-\widehat{w}\left(k_{0}\right)\left(\Phi_{1}|a|^{2}+\Phi_{4}|b|^{2}\right) a+\frac{\gamma}{2} b^{*}, \\
\left(1+g \widetilde{\eta}^{\prime}\left(-i \omega_{c}\right)\right) \frac{\partial b}{\partial t}=\Lambda b-\widehat{w}\left(k_{0}\right)\left(\Phi_{1}^{*}|b|^{2}+\Phi_{4}^{*}|a|^{2}\right) b+\frac{\gamma}{2} a^{*},
\end{gathered}
$$

where $\Lambda=\widehat{w}\left(k_{0}\right) \epsilon^{2} \delta+\beta_{c} \widehat{w}^{\prime \prime}\left(k_{0}\right)\left(i \partial_{x}+v_{1}\right)^{2} / 2$ and $\gamma=\epsilon^{2} \gamma_{2}$. We note that $\Phi_{4}$ is real when $k_{y}=$ 0 , but in general $\Phi_{1}$ is complex. Using the definition of $\widetilde{\eta}$ as in (3.6), and also the relationship between $g, \tau_{a}$ and the emergent frequency, $\omega_{c}$, of the dynamic pattern, the amplitude equations can be written in the form

$$
\begin{aligned}
& \frac{\partial a}{\partial t}=\frac{1}{2}\left(1-\frac{i}{\tau_{a} \omega_{c}}\right)\left(\Lambda a-\widehat{w}\left(k_{0}\right)\left(\Phi_{1}|a|^{2}+\Phi_{4}|b|^{2}\right) a+\frac{\gamma}{2} b^{*}\right), \\
& \frac{\partial b}{\partial t}=\frac{1}{2}\left(1+\frac{i}{\tau_{a} \omega_{c}}\right)\left(\Lambda b-\widehat{w}\left(k_{0}\right)\left(\Phi_{1}^{*}|b|^{2}+\Phi_{4}|a|^{2}\right) b+\frac{\gamma}{2} a^{*}\right) .
\end{aligned}
$$

We now look for spatially homogeneous solutions of (4.23)-(4.24), so take $\Lambda=\widehat{w}\left(k_{0}\right) \epsilon^{2} \delta+$ $\beta_{c} \widehat{w}^{\prime \prime}\left(k_{0}\right) v_{1}^{2} / 2$ which is also now real-valued. Writing $a=\rho_{a} \mathrm{e}^{i \phi_{a}}$ and $b=\rho_{b} \mathrm{e}^{i \phi_{b}}$ we find that 


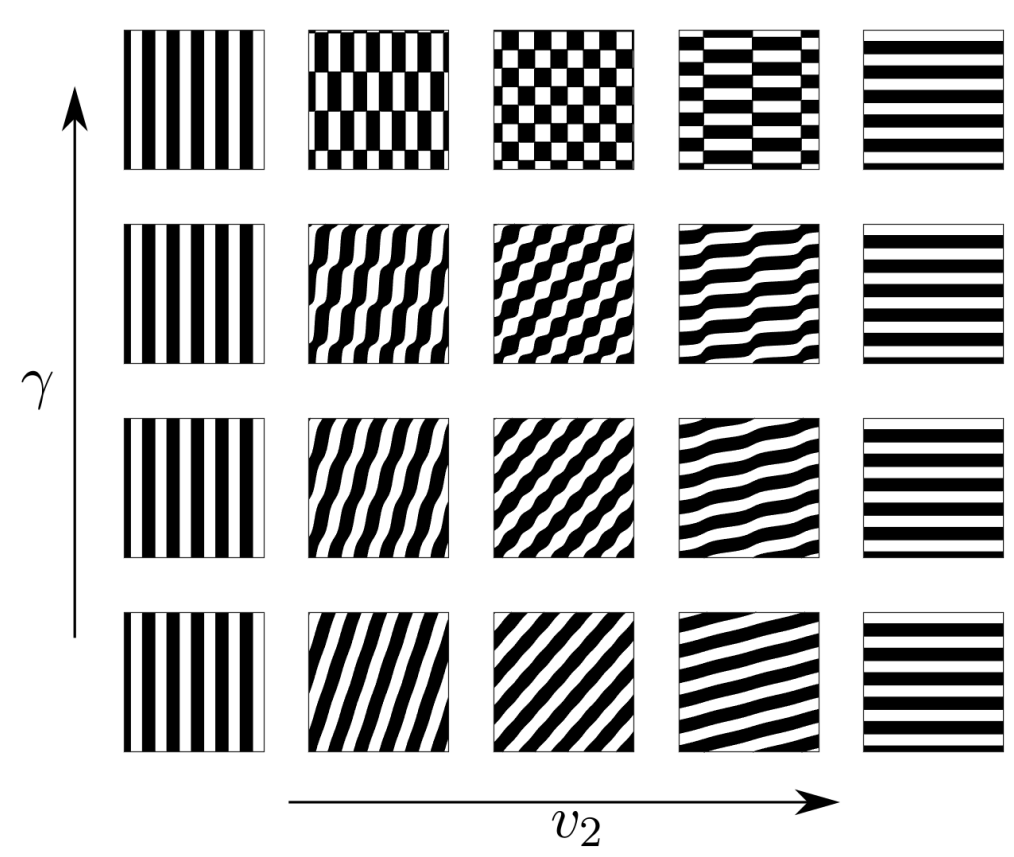

Figure 8: Planforms of the stable leading order solution demonstrating pattern diversity and orthogonal response. Choosing $h=0$ (so that existence and stability of solutions does not depend on $v_{2}$ ) we see that as $v_{2}$ is increased from 0 to $k_{0}$ the pattern changes from vertical stripes to rectangles (when $\gamma>\gamma_{c}$ ) or oblique patterns (when $\gamma<\gamma_{c}$ ) to horizontal stripes which are orthogonal to the forcing. This corresponds to varying $k_{x}$ from $k_{0}$ (with a response in the direction of forcing) to 0 (with a response orthogonal to the direction of forcing). Note that if we choose $h$ differently then for some values of $v_{2}$ these leading order solution patterns do not exist. Other parameter values are $\sigma=0.5, \epsilon^{2} \delta=0.3, v_{2}=[0,0.05,0.25,0.75,1] k_{0}$, $\gamma=[0.1,0.4,0.65,1.1]$. Planforms are plotted for $x, y \in[0,10 \pi]$.

the phases and amplitudes satisfy

$$
\begin{aligned}
\frac{\partial \rho_{a}}{\partial t}= & \frac{1}{2}\left(\Lambda \rho_{a}-\widehat{w}\left(k_{0}\right) \rho_{a}\left(\Phi_{1}^{r} \rho_{a}^{2}+\Phi_{4} \rho_{b}^{2}\right)+\frac{\gamma}{2} \rho_{b} \cos (\psi)\right) \\
& -\frac{1}{2 \tau_{a} \omega_{c}}\left(\frac{\gamma}{2} \rho_{b} \sin (\psi)+\widehat{w}\left(k_{0}\right) \Phi_{1}^{i} \rho_{a}^{3}\right),
\end{aligned}
$$

$$
\frac{\partial \rho_{b}}{\partial t}=\frac{1}{2}\left(\Lambda \rho_{b}-\widehat{w}\left(k_{0}\right) \rho_{b}\left(\Phi_{1}^{r} \rho_{b}^{2}+\Phi_{4} \rho_{a}^{2}\right)+\frac{\gamma}{2} \rho_{a} \cos (\psi)\right)
$$

$$
+\frac{1}{2 \tau_{a} \omega_{c}}\left(\frac{\gamma}{2} \rho_{a} \sin (\psi)-\widehat{w}\left(k_{0}\right) \Phi_{1}^{i} \rho_{b}^{3}\right)
$$

$$
\frac{\partial \psi}{\partial t}=-\frac{1}{2 \tau_{a} \omega_{c}}\left(\frac{\gamma}{2} \cos (\psi)\left(\frac{\rho_{b}}{\rho_{a}}-\frac{\rho_{a}}{\rho_{b}}\right)-\widehat{w}\left(k_{0}\right)\left(\rho_{a}^{2}-\rho_{b}^{2}\right)\left(\Phi_{1}^{r}-\Phi_{4}\right)\right)
$$

$$
-\frac{\gamma}{4} \sin (\psi)\left(\frac{\rho_{b}}{\rho_{a}}+\frac{\rho_{a}}{\rho_{b}}\right)-\frac{1}{2} \widehat{w}\left(k_{0}\right) \Phi_{1}^{i}\left(\rho_{a}^{2}-\rho_{b}^{2}\right),
$$

$$
\begin{aligned}
\frac{\partial \theta}{\partial t}= & -\frac{1}{2 \tau_{a} \omega_{c}}\left(2 \Lambda+\frac{\gamma}{2} \cos (\psi)\left(\frac{\rho_{b}}{\rho_{a}}+\frac{\rho_{a}}{\rho_{b}}\right)-\widehat{w}\left(k_{0}\right)\left(\rho_{a}^{2}+\rho_{b}^{2}\right)\left(\Phi_{1}^{r}+\Phi_{4}\right)\right) \\
& -\frac{\gamma}{4} \sin (\psi)\left(\frac{\rho_{b}}{\rho_{a}}-\frac{\rho_{a}}{\rho_{b}}\right)-\frac{1}{2} \widehat{w}\left(k_{0}\right) \Phi_{1}^{i}\left(\rho_{a}^{2}+\rho_{b}^{2}\right),
\end{aligned}
$$


where $\psi=\phi_{a}+\phi_{b}, \theta=\phi_{a}-\phi_{b}$ and $\Phi_{1}^{r}, \Phi_{1}^{i}$ denote the real and imaginary parts of $\Phi_{1}$ respectively.

Looking for solutions with constant and equal amplitudes $\rho_{a}=\rho_{b}=\rho_{0}$ we see that $\psi$ is constant when it takes the values $\psi=m \pi$ for $m=0,1$. Then

$$
\rho_{0}=\sqrt{\frac{\tau_{a} \omega_{c}\left(2 \Lambda+(-1)^{m} \gamma\right)}{2 \widehat{w}\left(k_{0}\right)\left(\tau_{a} \omega_{c}\left(\Phi_{1}^{r}+\Phi_{4}\right)+\Phi_{1}^{i}\right)}},
$$

and we observe that

$$
\begin{aligned}
\frac{\partial \theta}{\partial t} & =-\frac{1}{2 \tau_{a} \omega_{c}}\left(2 \Lambda+(-1)^{m} \gamma-2 \widehat{w}\left(k_{0}\right)\left(\Phi_{1}^{r}+\Phi_{4}-\tau_{a} \omega_{c} \Phi_{1}^{i}\right) \rho_{0}^{2}\right) \\
& =-\frac{1}{2 \tau_{a} \omega_{c}}\left(2 \Lambda+(-1)^{m} \gamma\right)\left(1+\tau_{a}^{2} \omega_{c}^{2}\right) \Phi_{1}^{i} .
\end{aligned}
$$

Therefore $\theta$ is constant when $\Phi_{1}^{i}=0$ corresponding to periodic standing wave solutions, and otherwise $\theta$ is a linear function of time, corresponding to amplitude modulated standing waves. Assuming that $\tau_{a} \omega_{c}\left(\Phi_{1}^{r}+\Phi_{4}\right)+\Phi_{1}^{i}>0$, the solution with $m=0$ exists when $\gamma>-2 \Lambda$ and the solution with $m=1$ exists for $\gamma<2 \Lambda$. Linear stability analysis shows that the solution with $\psi=m \pi$ is stable when

$$
(-1)^{m} \gamma>\max \left\{0, \frac{-2 \Lambda \tau_{a} \omega_{c}\left(\Phi_{1}^{r}-\Phi_{4}\right)}{2 \tau_{a} \omega_{c} \Phi_{1}^{r}+\Phi_{1}^{i}}, \frac{-2 \Lambda\left(\tau_{a} \omega\left(\Phi_{1}^{r}-\Phi_{4}\right)+\Phi_{1}^{i}\right)}{\tau_{a} \omega_{c}\left(3 \Phi_{1}^{r}+\Phi_{4}\right)+3 \Phi_{1}^{i}}\right\} .
$$

We note that $\Phi_{1}^{i}=0$ only when $h=0$ so that $\beta_{2}=0$ and in this case $\Phi_{4}=2 \Phi_{1}^{r}$. Therefore, in the case where $\Phi_{1}^{i}=0$, the solution with $\psi=m \pi$ is stable for $(-1)^{m} \gamma>2 \Lambda$.

We can also find stable solutions of (4.25)-(4.28) with unequal constant amplitudes. Suppose that $\psi$ takes the constant values $m \pi$ for $m=0,1$. Then from (4.27) we observe that either $\rho_{a}=\rho_{b}$ or

$$
\rho_{a} \rho_{b}=\frac{(-1)^{m} \gamma}{2 \widehat{w}\left(k_{0}\right)\left(\tau_{a} \omega_{c} \Phi_{1}^{i}-\Phi_{1}^{r}+\Phi_{4}\right)}:=P_{m} .
$$

In the latter case, substitution into (4.25) multiplied by $\rho_{a}$ reveals that the constant amplitudes have values $\rho_{a}=\rho_{ \pm}, \rho_{b}=\rho_{\mp}$ where $\left(\rho_{ \pm}\right)^{2}$ are the two roots of

$$
\widehat{w}\left(k_{0}\right) \Phi_{1}^{r} \rho^{4}-\left(\Lambda-\frac{\widehat{w}\left(k_{0}\right) \Phi_{1}^{i} P_{m}}{\tau_{a} \omega_{c}}\right) \rho^{2}-P_{m}\left(\frac{(-1)^{m} \gamma}{2}-\widehat{w}\left(k_{0}\right) \Phi_{4} P_{m}\right)=0 .
$$

Such solutions exist when the roots are real and positive. When $\Phi_{1}^{i}=0$ the solutions have constant $\theta=2 \phi_{a}-m \pi$ and therefore the solutions are periodic travelling waves. They exist when $\Lambda \Phi_{1}^{r}>0$ and for $|\gamma|<|\Lambda|$ and can also be shown to be stable in this parameter range (see Figure 9(a)). When $\Phi_{1}^{i} \neq 0$ the solutions have $\theta(t)=2 \phi_{a}(t)-m \pi$ and correspond to resonant amplitude modulated travelling waves. Numerical investigation with XPPAUT [9] for the parameter choices as in Figure 9 indicates that the solutions are stable wherever they exist. The stability region covers the range of values of forcing strength $\gamma$ where the modulated standing waves are unstable and there are also regions of bistability of the modulated standing 
and travelling waves. These solutions are indicated in Figure 9(b) in red $(m=0)$ and magenta $(m=1)$. We also find stable modulated travelling waves with constant $\rho_{a} \neq \rho_{b}$ and constant $\psi \neq 0$ as indicated in green in Figure 9(b). Figure 9 summarises the solution branches and their stability for $\Phi_{1}^{i}=0$ and $\Phi_{1}^{i} \neq 0$ respectively where other parameter values are as given in the caption. This indicates that travelling waves dominate for weak forcing, and there is an exchange of stability to standing waves for stronger forcing $\gamma$.

(a)

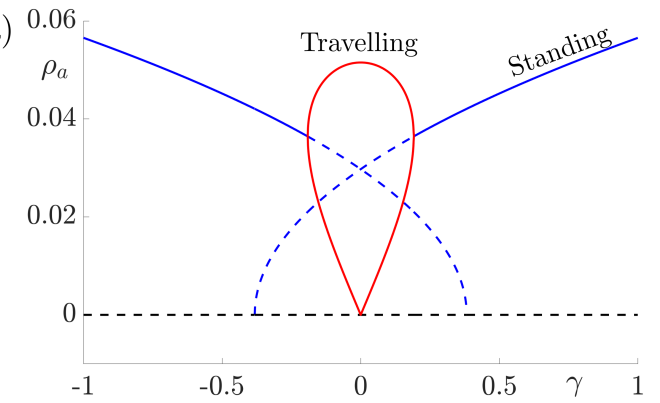

(b)

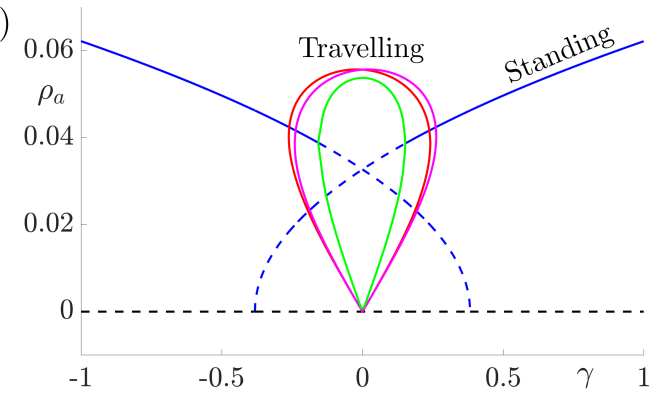

Figure 9: Bifurcation diagrams for resonant stripe pattern solutions for the forced neural field equation (3.12) in one spatial dimension with adaptation $(g \neq 0)$ under variation of the forcing strength $\gamma$. In (a) we take the threshold for the firing rate $h=0$ which gives $\Phi_{1}^{i}=0$ and therefore we observe periodic standing waves (blue) and travelling waves (red). Dashed lines indicate unstable solutions while solid lines indicate stable waves. In (b) we choose $|h|=0.05$ and therefore $\Phi_{1}^{i} \neq 0$ and we observe modulated (quasiperiodic) standing (blue) and various travelling (red, magenta and green) waves. Other parameter values for both diagrams are $\sigma=0.5, \tau_{a}=1, g=5$. These give $\beta_{c}=3, \widehat{w}\left(k_{0}\right)=2 / 3$ and $\widehat{w}^{\prime \prime}\left(k_{0}\right)=-16 / 27$ and here we take $\epsilon^{2} \delta=0.3$ and $v_{1}=0.1$ so that $\Lambda=\widehat{w}\left(k_{0}\right) \epsilon^{2} \delta+\beta_{c} \widehat{w}^{\prime \prime}\left(k_{0}\right) v_{1}^{2} / 2=43 / 225$.

The significant outcome of this investigation is that when adaptation is included, there are stable 2:1 resonant solutions which travel. Investigating the fully two-dimensional model with adaptation numerically reveals the same qualitative behaviour. Moreover, when the unforced system supports traveling waves, resonant rectangular patterns remain stationary but oblique patterns travel in an orthogonal direction, namely along the axis for which the continuous translational symmetry is not broken by the forcing. Thus, if spatial forcing is by a striped pattern along the $x$-direction then the tissue response could be a striped pattern in the orthogonal $y$-direction. Moreover, the presence of adaptation would allow for a dynamic instability so that this could propagate as a plane wave. Although the theory above has only been developed with spatially periodic forcing over the whole space, it has uncovered a mechanism for the generation of orthogonal responses that we expect to hold in the presence of more structured forcing. We explore this further in the next section and provide support for this claim using direct numerical simulations of forcing on the half-space relevant to the psychophysical experiments of Billock and Tsou [3].

5. Simulations and psychophysics. We now turn to the perception of patterns of activity in V1. One of the main structures of the visual cortex is that of retinotopy, a neurophysiological 
(a)

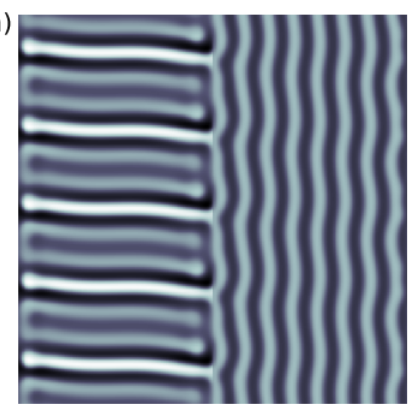

(c)

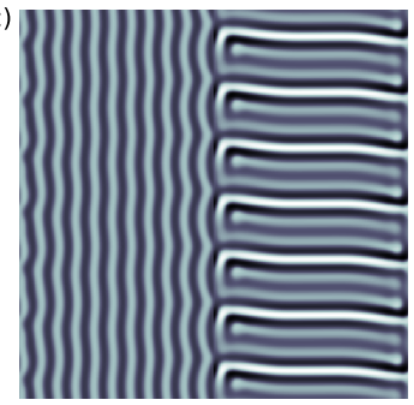

(b)

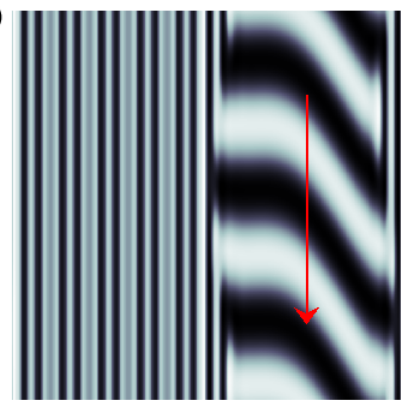

(d)

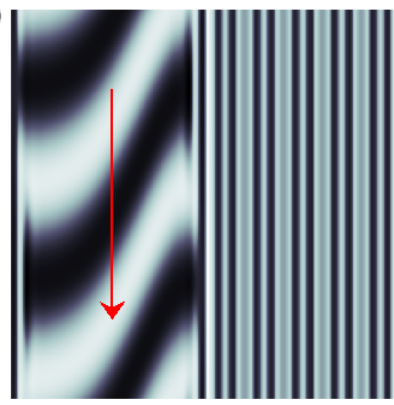

Figure 10: Simulation results from a neural field model with spatially periodic striped forcing on the half-space. (a) Horizontal stripes forcing the left half-space give rise to stationary vertical stripes on the right. (b) Vertical stripes forcing the left half-space give rise to travelling horizontal stripes on the right. (c) Horizontal stripes forcing the right half-space give rise to stationary vertical stripes on the left. (d) Vertical stripes forcing the right half-space give rise to travelling horizontal stripes on the left. An application of the inverse retinocortical map to (a), .., (d) generates patterns consistent with (a), ., (d) shown in Fig. 2. Parameter values are $\sigma=0.8, \mu=2, h=0.05, \gamma=0.5$ and for b) and d) $\tau_{a}=10, g=0.14$. The domain sizes are a) $[-16.53,16.53] \times[-15.71,15.71]$, b) $[-31.42,31.42] \times[-3.10,3.10]$, c) $[-22.73,22.73] \times[-22.00,22.00]$ and d) $[-31.42,31.42] \times[-2.07,2.07]$ with periodic boundary conditions. Movies available in Supplementary Materials.

projection of the retina to the visual cortex. The log-polar mapping [28] is perhaps the most common representation of the mapping of points from the retina to the visual cortex and see Fig. 2. The action of the retino-cortical map turns a circle of radius $r$ in the visual field into a vertical stripe at $x=\ln (r)$ in the cortex, and also turns a ray emanating from the origin with an angle $\theta$ into a horizontal stripe at $y=\theta$. Simply put, if a point on the visual 
field is described by $(r, \theta)$ in polar coordinates, the corresponding point in V1 has Cartesian coordinates $(x, y)=(\ln (r), \theta)$. Thus to answer how a pattern would be perceived we need only apply the inverse (conformal) log-polar mapping. The analytical work in previous sections has established that an orthogonal response to global spatially periodic forcing can be robustly supported in a standard neural field model. If the conditions for a resonant response are met, then a visual stimulus in the form of a set of concentric rings may give rise to a percept of a set of radial arms (one for each ring). Similarly, a visual stimulus in the form of a set of radial arms may give rise to a percept of a set of concentric rings. This is consistent with the observations of Billock and Tsou described in $\S 2$, albeit these are more accurately described by drive on the cortical half-space (since the stimuli do not cover the whole visual field). To complement our results for forcing on the whole cortical space we now turn to direct numerical simulations. By forcing with striped patterns on the cortical half-space we recover all of the features reported in Fig. 2, once the inverse retino-cortical map is applied. We show the corresponding plots for cortical activity in Fig. 10. The presence of the adaptation current allows the formation of travelling striped patterns, and these correspond to rotating waves in the retinal space with blinking versions associated to standing waves. Although the psychophysical experiments of Billock and Tsou involve a component of temporal flicker we have found that it is not strictly necessary to include this within the model to generate results consistent with their observations. Nonetheless, direct numerical simulations with flicker do show that the phenomenon is robust to this inclusion. We posit that in the psychophysical experiments the background flicker helps put the primary visual cortex in a state conducive to a 2:1 resonance, whereas in our model we tune intrinsic parameters to reach this condition.

Brief details of the numerical methods used to implement the model are presented in appendix C

6. Discussion. In this paper we have shown that the psychophysical observations of Billock and Tsou [3] can be explained with a parsimonious neural field model that does not require any exotic extension compared to standard approaches. It was originally suggested in [3, Supporting Information] that a neural field with some form of anisotropic coupling would be necessary to explain the observed spatial opponency between rings and radial arms. Rather we find, perhaps non-intuitively, that the pattern forming properties of a spatially forced isotropic model with a 2:1 resonance provide a sufficient mechanism for the observed phenonomena. Importantly, when the unforced model is poised near a Turing instability, we have shown that there are reasonably large windows of parameter space that allow for such a resonance between a spatial Turing pattern and a spatially periodic pattern of forcing. To establish this we have made use of perturbation arguments valid only for weak forcing. Nonetheless, this amplitude equation approach has proven especially useful for gaining insight into the main control parameters that can encourage an orthogonal response to the forcing of a two-dimensional neural field with a simple periodic stripe pattern. A key parameter in this regard is the deviation between $k_{0}$, the spatial frequency excited by the Turing instability, and $k_{f} / 2$, where $k_{f}$ is the spatial frequency of the forcing. An orthogonal response is promoted as this deviation becomes closer to $k_{0}$. As well as using mathematical arguments, strictly only valid for global periodic forcing, we have used direct numerical simulations to show that the model responds similarly when patterns are presented only on the half-space (which is more 
consistent with the psychophysical experiments). Moreover, we have shown that some form of negative feedback or adaptation is useful for promoting travelling Turing patterns, which (via the inverse retino-cortical map) generate rotating percepts. These would also be expected in a more refined two-population neural field model without adaptation that distinguishes between excitatory and inhibitory sub-populations [10,31]. We have opted for the study of an effective single population model with adaptation solely to keep the mathematical analysis manageable.

Here we have focused on the analysis of simple spatially repetitive and time-independent stimuli. Even simple variants of such patterns, such as the Enigma, created by pop-artist Isia Leviant [16], consisting of concentric annuli on top of a pattern of radial spokes, can lead to very striking illusory motion percepts. In future work we plan to consider input patterns with more spatial structure and explore the conditions for the emergence of global illusory percepts from local interactions, such as the Barber pole, Café wall, Fraser spiral, and Ehrenstein illusion in which local orientation differences lead to the appearance of the global rotation of contours (see [14] for further examples). Moreover, given that periodic and quasi-crystal patterns in physical (Faraday) systems can be excited by periodic temporal forcing [25] this motivates a further study of associated behaviour in neural models. It is known that fullfield flickering visual stimulation in humans can produce geometric hallucinations in the form of radial or spiral arms (and conversely that brain rhythms at the flicker frequency can be enhanced with the presentation of static radial or spiral arms) [23]. Indeed, flicker induced hallucinations have previously been studied from a theoretical perspective in neural fields with time periodic forcing by Rule et al. [26], and it would be very natural to extend the work here to include models of spatio-temporal sensory drive, and in particular to further understand visual hallucinations induced by flicker constrained to a thin annulus centred on the fovea [24]. Another natural extension is to extend very recent work on undriven neural fields that shows how quasi-crystal patterns can arise via a Turing instability [12] to further include spatio-temporal forcing. 


$$
\begin{aligned}
& \left\langle\mathrm{e}^{i k_{0} x}, \mathcal{L} u_{3}\right\rangle=0 \\
& \left\langle\mathrm{e}^{i k_{0} x}, \frac{\partial u}{\partial \tau}\right\rangle=\frac{\partial A}{\partial \tau}, \\
& \left\langle\mathrm{e}^{i k_{0} x}, 2 \beta_{2} w \otimes u_{1} u_{2}\right\rangle=2 \beta_{2} \widehat{w}\left(k_{0}\right) d_{0} A|A|^{2} \\
& \left\langle\mathrm{e}^{i k_{0} x}, \beta_{3} w \otimes u_{1}^{3}\right\rangle=3 \beta_{3} \widehat{w}\left(k_{0}\right) A|A|^{2}, \\
& \left\langle\mathrm{e}^{i k_{0} x}, \delta w \otimes u_{1}\right\rangle=\delta \widehat{w}\left(k_{0}\right) A \\
& \left\langle\mathrm{e}^{i k_{0} x}, \frac{1}{2} \beta_{c} W^{x x} \otimes \partial_{\chi \chi} u_{1}\right\rangle=\frac{1}{2} \beta_{c} \widehat{W}^{x x}\left(k_{0}\right) \frac{\partial^{2} A}{\partial \chi^{2}}, \\
& \left\langle\mathrm{e}^{i k_{0} x}, \beta_{2} W^{x} \otimes \partial_{\chi} u_{1}^{2}\right\rangle=0, \\
& \left\langle\mathrm{e}^{i k_{0} x}, \beta_{c} W^{x} \otimes \partial_{\chi} u_{2}\right\rangle=\left(1-\delta_{n, 2}\right) \frac{\overline{\gamma_{1}}}{2} \beta_{c} \widehat{W}^{x}\left(k_{0}\right) \alpha_{-} \mathrm{e}^{-2 i v x} \partial_{\chi} A^{*} \delta_{n, 2}=0, \\
& \left\langle\mathrm{e}^{i k_{0} x}, \gamma_{2} u_{1} \cos k_{f} x\right\rangle=\frac{\gamma_{2}}{2} A^{*} \mathrm{e}^{-2 i v x} \delta_{n, 2}=\frac{\gamma_{2}}{2} A^{*} \mathrm{e}^{-2 i c \chi} \delta_{n, 2}, \\
& \left\langle\mathrm{e}^{i k_{0} x}, \gamma_{1} u_{2} \cos k_{f} x\right\rangle=\left(1-\delta_{n, 2}\right)\left(\frac{\overline{\gamma_{1}}}{2}\right)^{2}\left[\left(d_{+}+d_{-}\right) A+\delta_{n, 1} d_{-} A^{*} \mathrm{e}^{-2 i v x}\right] \\
& \quad=\left(1-\delta_{n, 2}\right)\left(\frac{\bar{\gamma}_{1}}{2}\right)^{2}\left[\left(d_{+}+d_{-}\right) A+\delta_{n, 1} d_{-} A^{*} \mathrm{e}^{-2 i c \chi}\right] .
\end{aligned}
$$

Note further that

$$
\widehat{W}^{x}(k)=-\int_{-\infty}^{\infty} \mathrm{d} x \mathrm{e}^{-i k x} w(x) x=-i \frac{\mathrm{d}}{\mathrm{d} k} \int_{-\infty}^{\infty} \mathrm{d} x \mathrm{e}^{-i k x} w(x)=-i \frac{\mathrm{d}}{\mathrm{d} k} \widehat{w}(k) .
$$

Similarly $\widehat{W}^{x x}(k)=-\widehat{w}^{\prime \prime}(k)$. Also note that as $\widehat{w}\left(k_{0}\right)$ is a maximum its derivative is zero, so that $\widehat{W}^{x}\left(k_{0}\right)=0$.

\section{Appendix B. Derivation of amplitude equations for planar model with adaptation.}

Here we give details of the calculation of the amplitude equations (4.6)-(4.9) from the hierarchy of equations (4.2)-(4.4). We define an inner product of two functions which are spatially periodic with basic region $\Omega=\left[0,2 \pi / k_{x}\right] \times\left[0,2 \pi / k_{y}\right]$ and $2 \pi / \omega_{c}$ periodic in time as

$$
\langle U, V\rangle=\frac{\omega_{c}}{2 \pi|\Omega|} \int_{0}^{2 \pi / \omega_{c}} \int_{\Omega} U^{*}(\mathbf{r}, t) V(\mathbf{r}, t) \mathrm{d} \mathbf{r} \mathrm{d} t
$$

The hierarchy consists of equations of the form $\mathcal{L}_{g} u_{\alpha}=g_{\alpha}\left(u_{1}, \ldots, u_{\alpha}\right)$ for the linear operator $\mathcal{L}_{g}=-\frac{\partial}{\partial t}-1+\beta_{c} w \otimes-g \eta *$. The adjoint of this operator is $\mathcal{L}_{g}^{\dagger}=\frac{\partial}{\partial t}-1+\beta_{c} w \otimes-g \eta_{-} *$ where $\eta_{-}(t)=\eta(-t)$. For all $u \in \operatorname{ker} \mathcal{L}_{g}^{\dagger}$ then $\left\langle u, g_{\alpha}\right\rangle=\left\langle u, \mathcal{L}_{g} u_{\alpha}\right\rangle=\left\langle\mathcal{L}_{g}^{\dagger} u, u_{\alpha}\right\rangle=0$. It is straightforward to establish that $\operatorname{ker} \mathcal{L}_{g}^{\dagger}=\operatorname{ker} \mathcal{L}_{g}$ so that the set of solvability conditions are 
758

759

760

761

762

where

$\left\langle\mathrm{e}^{ \pm i\left(k_{x} x \pm k_{y} y \pm \omega_{c} t\right)}, g_{\alpha}\right\rangle=0$. We note that

$$
\begin{aligned}
& \left\langle\mathrm{e}^{i\left(k_{x} x+k_{y} y+\omega_{c} t\right)}, \mathcal{L}_{g} u_{2}\right\rangle=0, \quad\left\langle\mathrm{e}^{i\left(k_{x} x+k_{y} y+\omega_{c} t\right)}, \beta_{2} w \otimes u_{1}^{2}\right\rangle=0, \\
& \left\langle\mathrm{e}^{i\left(k_{x} x+k_{y} y+\omega_{c} t\right)}, \beta_{c}\left(W^{x} \otimes \partial_{\chi}+W^{y} \otimes \partial_{\Upsilon}\right) u_{1}\right\rangle=0, \\
& \left\langle\mathrm{e}^{i\left(k_{x} x+k_{y} y+\omega_{c} t\right)}, \gamma_{1} u_{1} \cos k_{f} x\right\rangle=\left\{\begin{array}{ll}
0 & n \neq 2 \\
\frac{\gamma_{1}}{2} A_{4}^{*} \mathrm{e}^{-2 i v_{1} x} & n=2
\end{array},\right.
\end{aligned}
$$

and hence the solvability condition is automatically satisfied for all $n \neq 2$ and for $n=2$ we must set $\gamma_{1}=0$. We write $\gamma_{1}=\left(1-\delta_{n, 2}\right) \bar{\gamma}_{1}$. We find a particular solution $u_{2}$ by assuming that it has the form of $u_{1}^{2}+\left(1-\delta_{n, 2}\right) \bar{\gamma}_{1} u_{1} \cos \left(k_{f} x\right)$, substituting into (4.3) and balancing terms. For our balanced kernel where $\widehat{w}(0)=0$ we find that

$$
\begin{gathered}
u_{2}=\zeta_{1}\left(A_{1}^{2} \mathrm{e}^{2 i\left(k_{x} x+k_{y} y+\omega_{c} t\right)}+A_{2}^{2} \mathrm{e}^{2 i\left(k_{x} x-k_{y} y+\omega_{c} t\right)}+\left(A_{3}^{*}\right)^{2} \mathrm{e}^{-2 i\left(k_{x} x+k_{y} y-\omega_{c} t\right)}\right. \\
\left.+\left(A_{4}^{*}\right)^{2} \mathrm{e}^{-2 i\left(k_{x} x-k_{y} y-\omega_{c} t\right)}\right)+\zeta_{2}\left(A_{1} A_{2} \mathrm{e}^{2 i\left(k_{x} x+\omega_{c} t\right)}+A_{3}^{*} A_{4}^{*} \mathrm{e}^{-2 i\left(k_{x} x-\omega_{c} t\right)}\right) \\
+\zeta_{3}\left(A_{1} A_{3} \mathrm{e}^{2 i\left(k_{x} x+k_{y} y\right)}+A_{2} A_{4} \mathrm{e}^{2 i\left(k_{x} x-k_{y} y\right)}\right)+\zeta_{4}\left(A_{1} A_{4}+A_{2} A_{3}\right) \mathrm{e}^{2 i k_{x} x} \\
+\zeta_{5}\left(A_{1} A_{2}^{*}+A_{3} A_{4}^{*}\right) \mathrm{e}^{2 i k_{y} y}+\zeta_{6}\left(A_{1} A_{4}^{*} \mathrm{e}^{2 i\left(k_{y} y+\omega_{c} t\right)}+A_{2} A_{3}^{*} \mathrm{e}^{-2 i\left(k_{y} y-\omega_{c} t\right)}\right) \\
\frac{\bar{\gamma}_{1}}{2}\left(1-\delta_{n, 2}\right)\left[\zeta _ { + } \left(A_{1} \mathrm{e}^{i\left(\left(k_{x}+k_{f}\right) x+k_{y} y+\omega_{c} t\right)}+A_{2} \mathrm{e}^{i\left(\left(k_{x}+k_{f}\right) x-k_{y} y+\omega_{c} t\right)}+A_{3}^{*} \mathrm{e}^{-i\left(\left(k_{x}+k_{f}\right) x+k_{y} y-\omega_{c} t\right)}\right.\right. \\
\left.+A_{4}^{*} \mathrm{e}^{-i\left(\left(k_{x}+k_{f}\right) x-k_{y} y-\omega_{c} t\right)}\right) \\
+\zeta_{-}\left(A_{1} \mathrm{e}^{i\left(\left(k_{x}-k_{f}\right) x+k_{y} y+\omega_{c} t\right)}+A_{2} \mathrm{e}^{i\left(\left(k_{x}-k_{f}\right) x-k_{y} y+\omega_{c} t\right)}+A_{3}^{*} \mathrm{e}^{-i\left(\left(k_{x}-k_{f}\right) x+k_{y} y-\omega_{c} t\right)}\right. \\
\left.\left.+A_{4}^{*} \mathrm{e}^{-i\left(\left(k_{x}-k_{f}\right) x-k_{y} y-\omega_{c} t\right)}\right)\right]+ \text { c.c. },
\end{gathered}
$$

$$
\zeta_{1}=\frac{\beta_{2} \widehat{w}\left(2 k_{0}\right)}{2 i \omega_{c}+1-\beta_{c} \widehat{w}\left(2 k_{0}\right)+g \widetilde{\eta}\left(2 i \omega_{c}\right)}, \quad \zeta_{2}=\frac{2 \beta_{2} \widehat{w}\left(2 k_{x}\right)}{2 i \omega_{c}+1-\beta_{c} \widehat{w}\left(2 k_{x}\right)+g \widetilde{\eta}\left(2 i \omega_{c}\right)},
$$

$$
\zeta_{3}=\frac{2 \beta_{2} \widehat{w}\left(2 k_{0}\right)}{1-\beta_{c} \widehat{w}\left(2 k_{0}\right)+g}, \quad \zeta_{4}=\frac{2 \beta_{2} \widehat{w}\left(2 k_{x}\right)}{1-\beta_{c} \widehat{w}\left(2 k_{x}\right)+g},
$$

$$
\begin{aligned}
\zeta_{5}=\frac{2 \beta_{2} \widehat{w}\left(2 k_{y}\right)}{1-\beta_{c} \widehat{w}\left(2 k_{y}\right)+g}, & \zeta_{6}=\frac{2 \beta_{2} \widehat{w}\left(2 k_{y}\right)}{2 i \omega_{c}+1-\beta_{c} \widehat{w}\left(2 k_{y}\right)+g \widetilde{\eta}\left(2 i \omega_{c}\right)}, \\
\zeta_{ \pm}=\frac{1}{i \omega_{c}+1-\beta_{c} \widehat{w}\left(k_{ \pm}\right)+g \widetilde{\eta}\left(i \omega_{c}\right)}, & k_{ \pm}=\sqrt{\left(k_{x} \pm k_{f}\right)^{2}+k_{y}^{2}} .
\end{aligned}
$$


782 We now use this in the solvability conditions for $\alpha=3$ where we find the following projections;

$$
\begin{aligned}
& 783 \text { (B.10) }\left\langle\mathrm{e}^{i\left(k_{x} x+k_{y} y+\omega_{c} t\right)}, \mathcal{L}_{g} u_{3}\right\rangle=0 \text {, } \\
& 784 \text { (B.11) }\left\langle\mathrm{e}^{i\left(k_{x} x+k_{y} y+\omega_{c} t\right)}, \partial_{\tau} u_{1}\right\rangle=\frac{\partial A_{1}}{\partial \tau}, \\
& 785 \text { (B.12) }\left\langle\mathrm{e}^{i\left(k_{x} x+k_{y} y+\omega_{c} t\right)}, \delta w \otimes u_{1}\right\rangle=\delta \widehat{w}\left(k_{0}\right) A_{1} \text {, } \\
& 786 \\
& \left\langle\mathrm{e}^{i\left(k_{x} x+k_{y} y+\omega_{c} t\right)}, \beta_{c} W^{x y} \otimes \partial_{\chi} \Upsilon u_{1}\right\rangle=-\beta_{c}\left(\widehat{w}^{\prime}\left(k_{0}\right)\right)^{2} \frac{\partial^{2} A_{1}}{\partial \chi \partial \Upsilon}=0, \\
& \left\langle\mathrm{e}^{i\left(k_{x} x+k_{y} y+\omega_{c} t\right)}, \frac{\beta_{c}}{2} W^{y y} \otimes \partial \Upsilon \Upsilon u_{1}\right\rangle=-\frac{\beta_{c}}{2} \widehat{w}^{\prime \prime}\left(k_{0}\right) \frac{\partial^{2} A_{1}}{\partial \Upsilon^{2}}, \\
& \left\langle\mathrm{e}^{i\left(k_{x} x+k_{y} y+\omega_{c} t\right)}, \beta_{2} W^{x} \otimes \partial_{\chi} u_{1}^{2}\right\rangle=0 \\
& \left\langle\mathrm{e}^{i\left(k_{x} x+k_{y} y+\omega_{c} t\right)}, \beta_{c} W^{x} \otimes \partial_{\chi} u_{2}\right\rangle=0, \\
& \left\langle\mathrm{e}^{i\left(k_{x} x+k_{y} y+\omega_{c} t\right)}, g \eta^{t} * \partial_{\tau} u_{1}\right\rangle=g \widetilde{\eta}^{\prime}\left(i \omega_{c}\right) \frac{\partial A_{1}}{\partial \tau}, \\
& \left\langle\mathrm{e}^{i\left(k_{x} x+k_{y} y+\omega_{c} t\right)}, \bar{\gamma}_{1}\left(1-\delta_{n, 2}\right) u_{2} \cos \left(k_{f} x\right)\right\rangle \\
& =\left(\frac{\bar{\gamma}_{1}}{2}\right)^{2}\left(1-\delta_{n, 2}\right)\left[\left(\zeta_{+}+\zeta_{-}\right) A_{1}+\zeta_{-} A_{4}^{*} \mathrm{e}^{-2 i v_{1} x} \delta_{n, 1}\right] \text {, } \\
& \left\langle\mathrm{e}^{i\left(k_{x} x+k_{y} y+\omega_{c} t\right)}, \gamma_{2} u_{1} \cos \left(k_{f} x\right)\right\rangle=\frac{\gamma_{2}}{2} A_{4}^{*} \mathrm{e}^{-2 i v_{1} x} \delta_{n, 2} \text {. }
\end{aligned}
$$

Here, we note that

We also have the scaling $v_{1}=\epsilon c_{1}$ so $\mathrm{e}^{-2 i v_{1} x}=\mathrm{e}^{-2 i c_{1} \chi}$. The projections give the evolution of the amplitude $A_{1}$ as

$$
\begin{aligned}
\left(1+g \widetilde{\eta}^{\prime}\left(i \omega_{c}\right)\right) \frac{\partial A_{1}}{\partial \tau} & =\widehat{w}\left(k_{0}\right)\left(\delta A_{1}-\sum_{j=1}^{4} \Phi_{j}\left|A_{j}\right|^{2} A_{1}-\Phi_{5} A_{2} A_{3} A_{4}^{*}\right) \\
& -\frac{\beta_{c}}{2} \widehat{w}^{\prime \prime}\left(k_{0}\right)\left(\frac{\partial^{2} A_{1}}{\partial \chi^{2}}+\frac{\partial^{2} A_{1}}{\partial \Upsilon^{2}}\right)+\frac{\gamma_{2}}{2} A_{4}^{*} \mathrm{e}^{-2 i c_{1} \chi} \delta_{n, 2} \\
& +\left(\frac{\bar{\gamma}_{1}}{2}\right)^{2}\left(1-\delta_{n, 2}\right)\left[\left(\zeta_{+}+\zeta_{-}\right) A_{1}+\zeta_{-} A_{4}^{*} \mathrm{e}^{-2 i c_{1} \chi} \delta_{n, 1}\right]
\end{aligned}
$$


where

$$
\begin{aligned}
& \Phi_{1}=-2 \beta_{2} \zeta_{1}-3 \beta_{3}, \quad \Phi_{2}=-2 \beta_{2}\left(\zeta_{2}+\zeta_{5}\right)-6 \beta_{3}, \quad \Phi_{3}=-2 \beta_{2} \zeta_{3}-6 \beta_{3}, \\
& \Phi_{4}=-2 \beta_{2}\left(\zeta_{4}+\zeta_{6}\right)-6 \beta_{3}, \quad \Phi_{5}=-2 \beta_{2}\left(\zeta_{4}+\zeta_{5}\right)-6 \beta_{3},
\end{aligned}
$$

and we note that $\widetilde{\eta}^{\prime}\left(-i \omega_{c}\right)=\left(\widetilde{\eta}^{\prime}\left(i \omega_{c}\right)\right)^{*}$. Similarly, by considering the projections $\left\langle\mathrm{e}^{i\left(k_{x} x-k_{y} y+\omega_{c} t\right)}, \cdot\right\rangle,\left\langle\mathrm{e}^{i\left(k_{x} x+k_{y} y-\omega_{c} t\right)}, \cdot\right\rangle$ and $\left\langle\mathrm{e}^{i\left(k_{x} x-k_{y} y-\omega_{c} t\right)}, \cdot\right\rangle$ we find the corresponding evolution equations for the amplitudes $A_{2}, A_{3}$ and $A_{4}$ respectively. Note that away from the bifurcation the solution will have a (temporal) frequency $\omega=\omega_{c}+\xi$ where $\xi$ is a frequency detuning parameter which we can assume is order $\epsilon^{2}$. Recall also that we also have the spatial frequency detuning parameter $v_{1}=k_{x}-k_{f} / n$. When we rescale back to the original length and timescales we also let $a_{j}=\epsilon A_{j} \mathrm{e}^{i c_{1} \chi} \mathrm{e}^{i \xi_{j} t}, j=1,2,3,4$ where $\xi_{1}=\xi_{2}=-\xi$ and $\xi_{3}=\xi_{4}=\xi$. Upon rescaling

$$
\frac{\partial A_{j}}{\partial \tau} \rightarrow \frac{\mathrm{e}^{-i v_{1} x}}{\epsilon^{3}} \frac{\partial}{\partial t}\left(a_{j} \mathrm{e}^{-i \xi_{j} t}\right)=\frac{\mathrm{e}^{-i v_{1} x} \mathrm{e}^{-i \xi_{j} t}}{\epsilon^{3}}\left(\frac{\partial a_{j}}{\partial t}-i \xi_{j} a_{j}\right) .
$$

The parameter $\xi$ can be removed from the amplitude equations by noting that the factor outside the bracket in (B.27) is also a factor on the right hand side of the rescaled amplitude equation and by making a transformation $a_{j} \rightarrow a_{j} \mathrm{e}^{i \xi_{j} t}$. The transformation removes the imaginary term inside the bracket and is equivalent to changing the carrier wave frequency to $\omega=\omega_{c}+\xi$. The resulting amplitude equations are (4.6)-(4.9).

\section{Appendix C. Numerical methods.}

The numerical simulation of the full model (2.1)-(2.2) were performed in the plane by discretising in space on a regular square mesh, and solving the resultant set of ordinary differential equations using MATLAB. A pseudo-spectral evaluation of the convolution $w \otimes f(u)$ was performed using a Fast Fourier Transform (FFT), followed by an inverse FFT on a large square computational domain. The Fourier transform of $w \otimes f$ takes the product form $\widehat{w} \times \widehat{f}$, and this provides substantial computational speed-up over quadrature-based numerical methods for calculating $w \otimes f(u)$. We set a grid of $N=2^{8}$ equally spaced points along each spatial dimension, and used MATLAB's in-built ode45 algorithm to evolve the system forward in time.

\section{REFERENCES}

[1] S. Amari, Dynamics of pattern formation in lateral-inhibition type neural fields, Biological Cybernetics, 27 (1977), pp. 77-87.

[2] M. Balasubramanian, J. Polimeni, and E. L. Schwartz, The V1-V2-V3 complex: Quasiconformal dipole maps in primate striate and extra-striate cortex, Neural Networks, 15 (2002), pp. 1157-1163.

[3] V. A. Billock AND B. H. Tsou, Neural interactions between flicker-induced self-organized visual hallucinations and physical stimuli, Proceedings of the National Academy of Sciences, 104 (2007), pp. 84908495.

[4] P. C. Bressloff, J. D. Cowan, M. Golubitsky, P. J. Thomas, and M. Wiener, Geometric visual hallucinations, Euclidean symmetry and the functional architecture of striate cortex, Philosophical Transactions of the Royal Society London B, 40 (2001), pp. 299-330.

[5] B. R. Conway, A. Kitaoka, A. Yazdanbakhsh, C. C. PaCk, and M. S. Livingstone, Neural basis for a powerful static motion illusion, Journal of Neuroscience, 25 (2005), pp. 5651-5656. 
[6] S. Coombes, P. Beim Graben, R. Potthast, and J. Wright, eds., Neural Fields: Theory and Applications, Springer, 2014.

[7] R. Curtu And B. Ermentrout, Pattern formation in a network of excitatory and inhibitory cells with adaptation, SIAM J. Applied Dynamical Systems, 3 (2004), pp. 191-231.

[8] G. B. Ermentrout, Neural networks as spatio-temporal pattern-forming systems, Reports on Progress in Physics, 61 (1998), pp. 353-430.

[9] G. B. Ermentrout, Simulating, analyzing, and animating dynamical systems: A guide to XPPAUT for researchers and students, SIAM Books, Philadelphia, 2002.

[10] G. B. Ermentrout And J. D. Cowan, A mathematical theory of visual hallucination patterns, Biological Cybernetics, 34 (1979), pp. 137-150.

[11] J. M. Gambaudo, Perturbation of a Hopf bifurcation by an external time-periodic forcing, Journal of Differential Equations, 57 (1985), pp. 172-199.

[12] A. Gökçe, D. Avitabile, and S. Coombes, Quasicrystal patterns in a neural field model, Physical Review Research, 2 (2020), p. 013234.

[13] A. Johnston, A spatial property of the retino-cortical mapping, Spatial Vision, 1 (1986), pp. 319-331.

[14] A. KitaOKA, Akiyoshi's illusion pages, http://www.ritsumei.ac.jp/ akitaoka/index-e.html.

[15] H. Kluver, Mescal and Mechanisms of Hallucinations, University of Chicago Press, Chicago, 1966.

[16] I. LÉviant, Illusory Motion within Still Pictures: The L-Effect, Leonardo, 15 (1982), pp. 222-223.

[17] A. L. Lin, A. Hagberg, E. Meron, And H. L. Swinney, Resonance tongues and patterns in periodically forced reaction-diffusion systems, Physical Review E, 69 (2004), p. 066217.

[18] D. M. MACKAY, Moving visual images produced by regular stationary patterns, Nature, 180 (1957), pp. 849-850.

[19] R. Manor, A. Hagberg, And E. Meron, Wave-number locking in spatially forced pattern-forming systems, EPL (Europhysics Letters), 83 (2008), p. 10005.

[20] R. Manor, A. Hagberg, And E. Meron, Wavenumber locking and pattern formation in spatially forced systems, New Journal of Physics, 11 (2009), p. 63016.

[21] Y. Mau, A. Hagberg, and E. Meron, Spatial Periodic Forcing Can Displace Patterns It Is Intended to Control, Physical Review Letters, 034102 (2012), pp. 1-5.

[22] Y. Mau, L. Haim, A. Hagberg, and E. Meron, Competing resonances in spatially forced patternforming systems, Physical Review E, 88 (2013), pp. 1-9.

[23] F. Mauro, A. Raffone, and R. VanRullen, A bidirectional link between brain oscillations and geometric patterns, Journal of Neuroscience, 35 (2015), pp. 7921-7926.

[24] J. Pearson, R. Chiou, S. Rogers, M. Wicken, S. Heitmann, and B. Ermentrout, Sensory dynamics of visual hallucinations in the normal population, eLife, 5 (2016), p. e17072.

[25] A. M. Rucklidge And M. Silber, Quasipatterns in parametrically forced systems, Physical Review E, 75 (2007), p. 055203.

[26] M. Rule, M. Stoffregen, and B. Ermentrout, A model for the origin and properties of flickerinduced geometric phosphenes, PLOS Computational Biology, 7 (2011), pp. 1-14.

[27] E. Schwartz, Spatial mapping in the primate sensory projection: analytic structure and relevance to projection, Biological Cybernetics, 25 (1977), pp. 181-194.

[28] E. L. Schwartz, Computational anatomy and functional architecture of striate cortex: A spatial mapping approach to perceptual coding, Vision Research, 20 (1980), pp. 645-669.

[29] R. Sokoliuk And R. VAnRullen, The flickering wheel illusion: when a rhythms make a static wheel flicker, Journal of Neuroscience, 33 (2013), pp. 13498-13504.

[30] P. TAss, Cortical pattern formation during visual hallucinations, Journal of Biological Physics, 21 (1995), pp. 177-210.

[31] P. TASs, Oscillatory cortical activity during visual hallucinations, Journal of Biological Physics, 23 (1997), pp. $21-66$. 